Supporting Information for:

\title{
Correlating Mechanical Sensitivity with Spin Transition in the Explosive Spin Crossover Complex $\left[\mathrm{Fe}(\mathrm{Htrz})_{3}\right]_{n}\left[\mathrm{ClO}_{4}\right]_{2 n}$
}

Thuy-Ai D. Nguyen, ${ }^{*}{ }^{\dagger}$ Jacqueline M. Veauthier, ${ }^{*},{ }^{\dagger}$ Gary F. Angles-Tamayo,,$^{\dagger}$ David E. Chavez, ${ }^{\dagger}$ Ekaterina Lapsheva, ${ }^{\ddagger}$ Thomas W. Myers,,$^{\dagger}$ Tammie R. Nelson,$^{\dagger}$ Eric J. Schelter ${ }^{\ddagger}$

${ }^{\dagger}$ Explosive Science and Shock Physics, Chemistry, and Theoretical Divisions, Los Alamos National Laboratory

${ }^{7}$ Department of Chemistry, University of Pennsylvania

\author{
*tdnguyen@lanl.gov \\ *veauthier@lanl.gov
}




\section{Table of Contents}

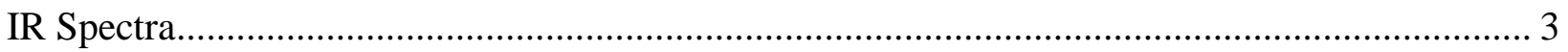

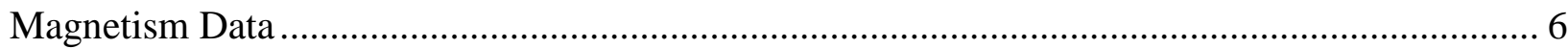

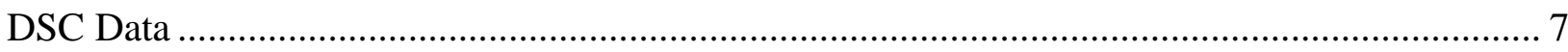

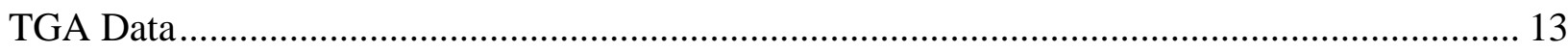

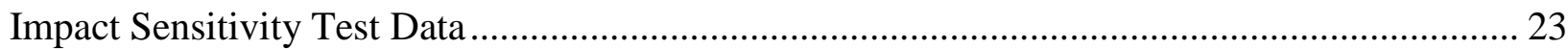

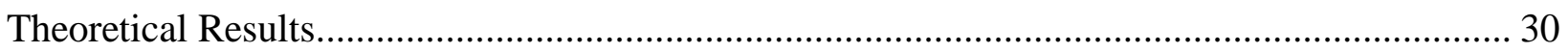




\section{IR Spectra}

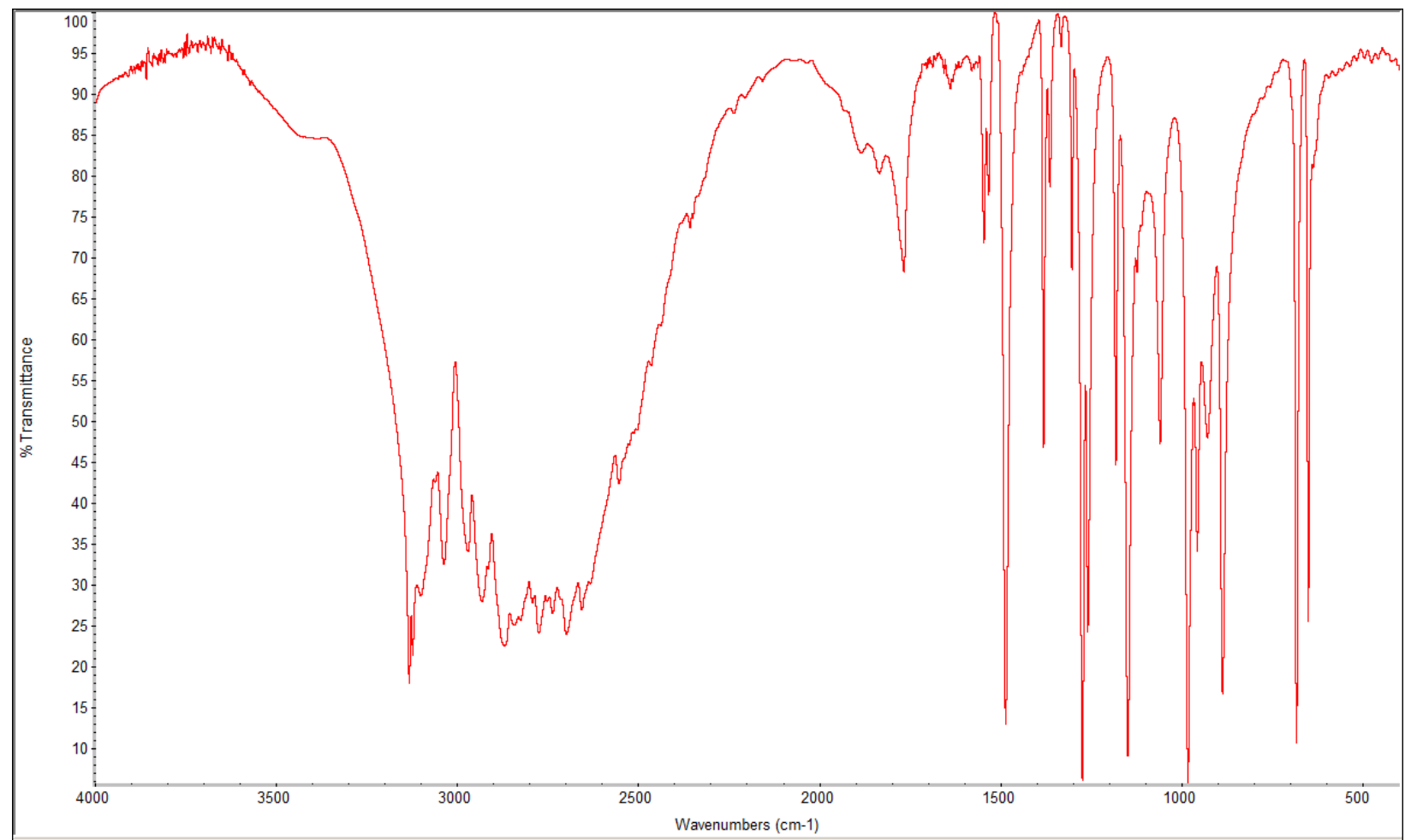

Figure S1. IR spectrum of 1,2,4-triazole (Htrz) (KBr pellet). Htrz IR (cm $\left.{ }^{-1}\right): 649$ (s), 682 (s), 885 (s), $927(\mathrm{~m}), 955(\mathrm{~m}), 981(\mathrm{~s}), 1058(\mathrm{~m}), 1147$ (s), $1180(\mathrm{~m}), 1257$ (s), 1272 (s), $1301(\mathrm{w}), 1362$ (w), 1379 (m), $1484(\mathrm{~s}), 1531(\mathrm{w}), 1544(\mathrm{w}), 1765(\mathrm{w}), 1832(\mathrm{w}), 1883(\mathrm{w}), 2355(\mathrm{w}), 2551(\mathrm{~m})$, 2654 (s), 2696 (s), 2734 (s), 2772 (s), 2867 (s), 2929 (s), 2967 (m), 3034 (m), 3098 (s), 3120 (s), 3129 (s). 


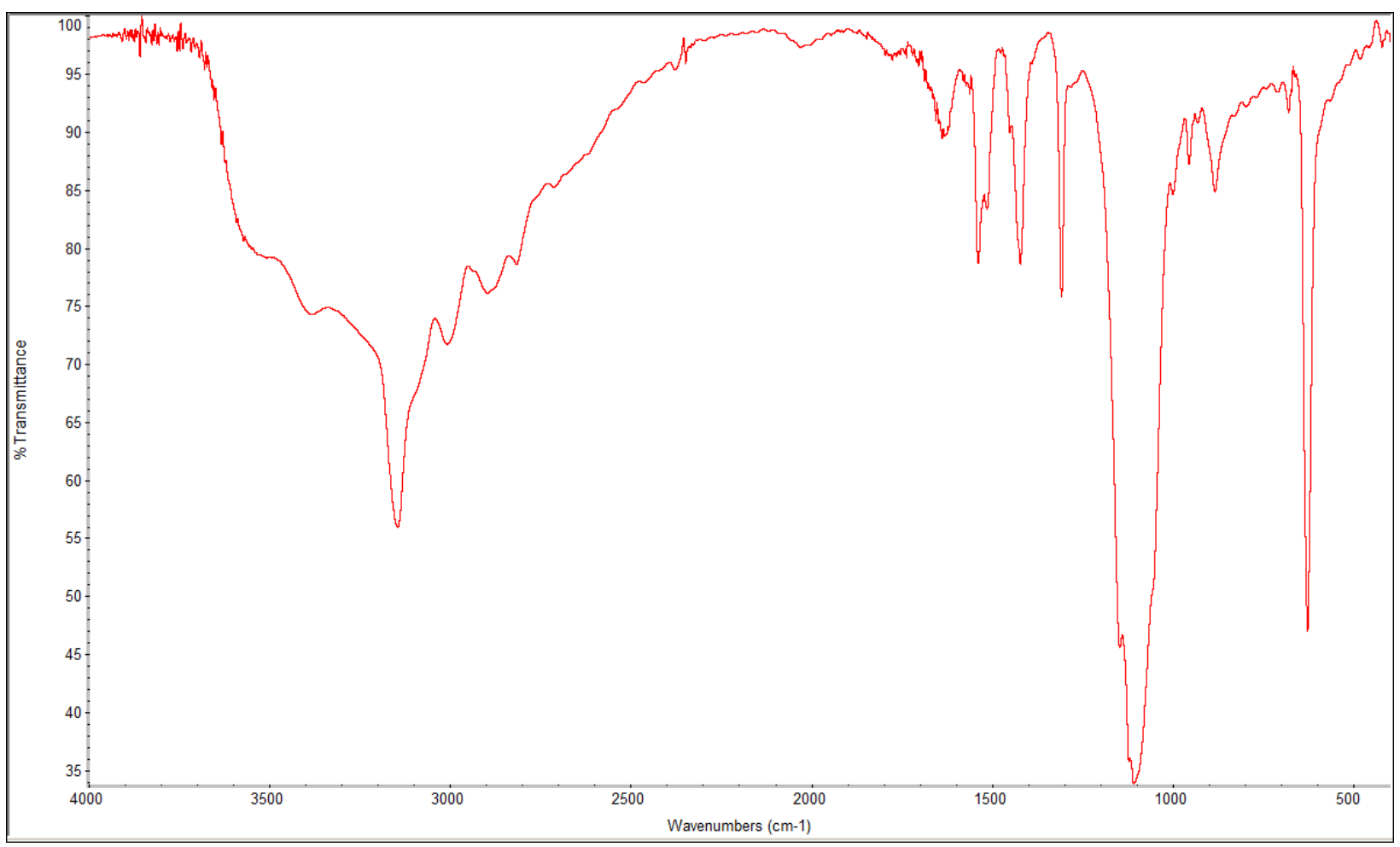

Figure S2. IR spectrum of $\left[\mathrm{Fe}(\mathrm{Htrz})_{3}\right]_{n}\left[\mathrm{ClO}_{4}\right]_{2 n}(\mathbf{1})(\mathrm{KBr}$ pellet).

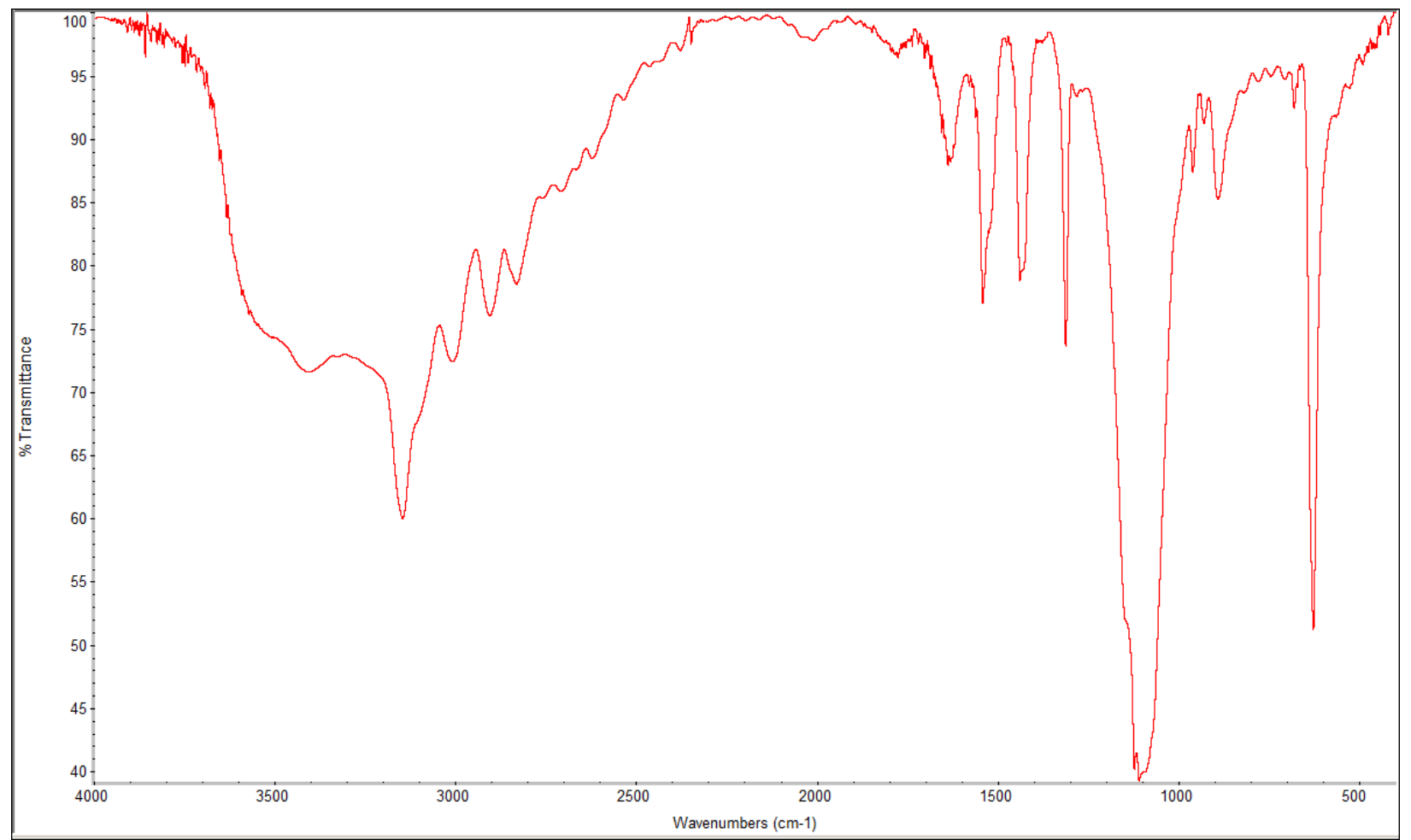

Figure S3. IR spectrum of $\left[\mathrm{Ni}(\mathrm{Htrz})_{3}\right]_{n}\left[\mathrm{ClO}_{4}\right]_{2 n}(\mathbf{2})$ (KBr pellet). 


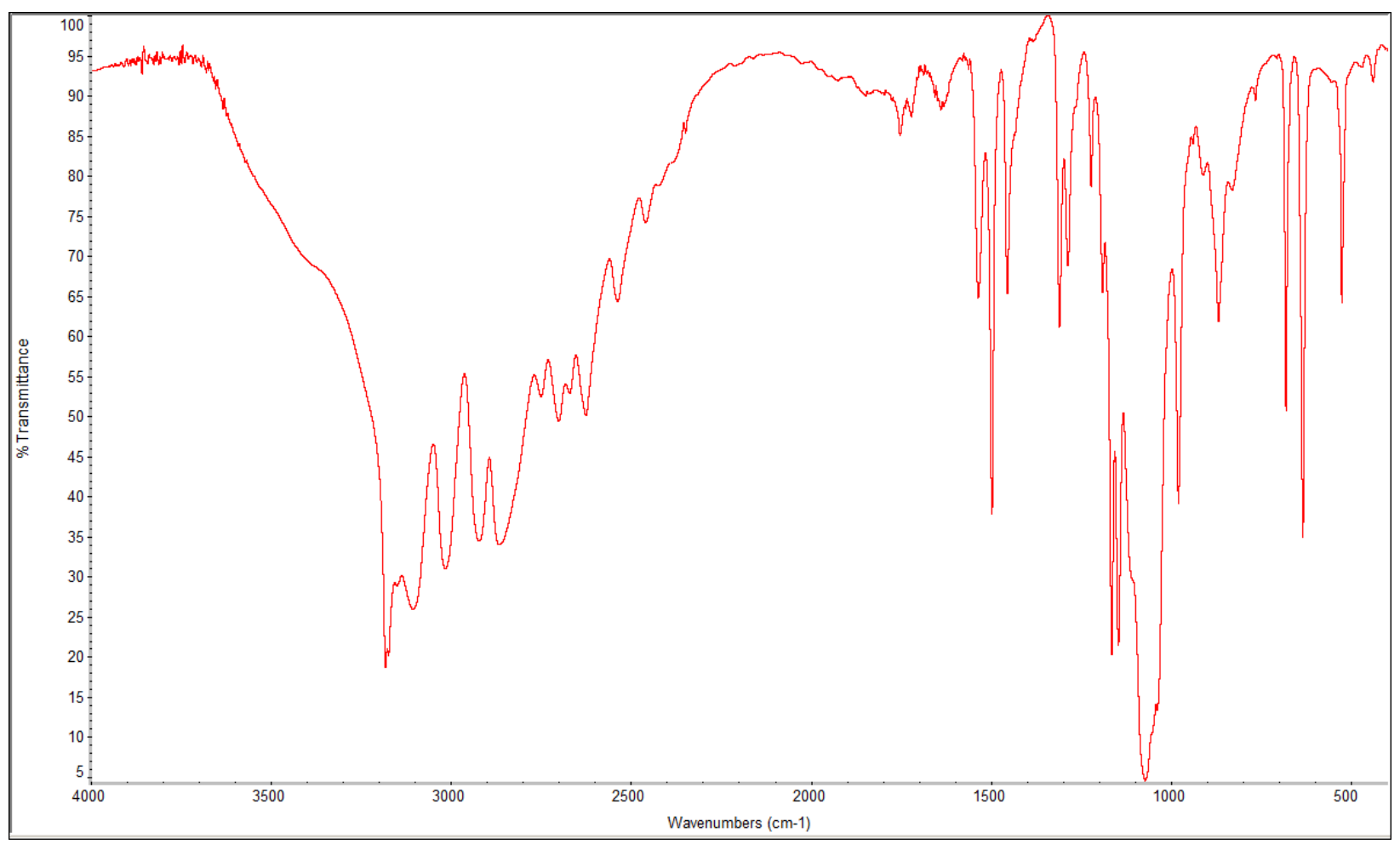

Figure S4. IR spectrum of $\left[\mathrm{Fe}(\mathrm{Htrz})_{2}(\mathrm{trz})\right]_{n}\left[\mathrm{BF}_{4}\right]_{n}(\mathbf{3})(\mathrm{KBr}$ pellet$)$. 


\section{Magnetism Data}

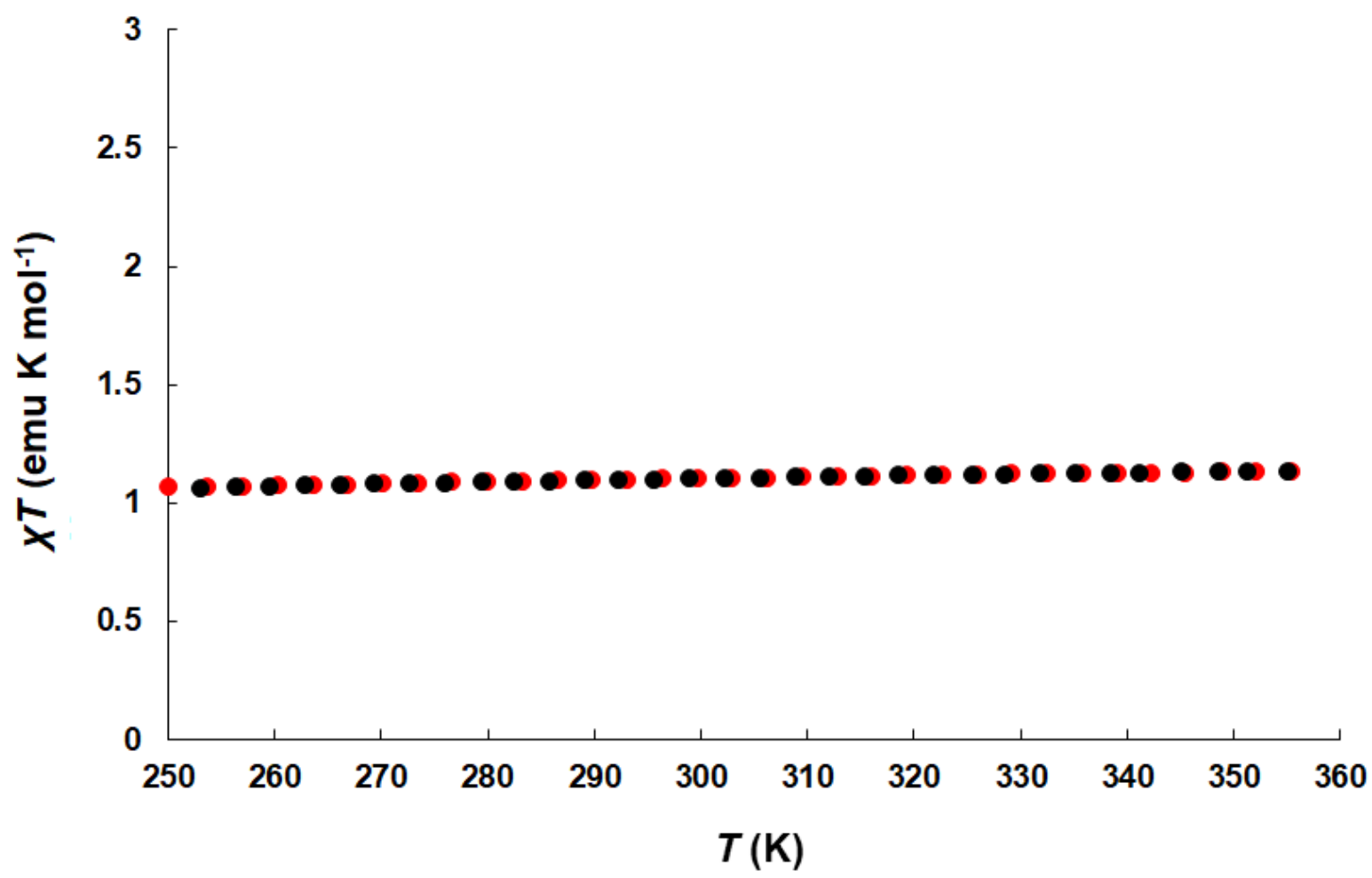

Figure S5. Temperature dependence of the $\chi \mathrm{T}$ product of $\left[\mathrm{Ni}(\mathrm{Htrz})_{3}\right]_{n}\left[\mathrm{ClO}_{4}\right]_{2 n}$ (2) from 250 to $355 \mathrm{~K}$ interval in $1 \mathrm{~T}$ field. 


\section{DSC Data}

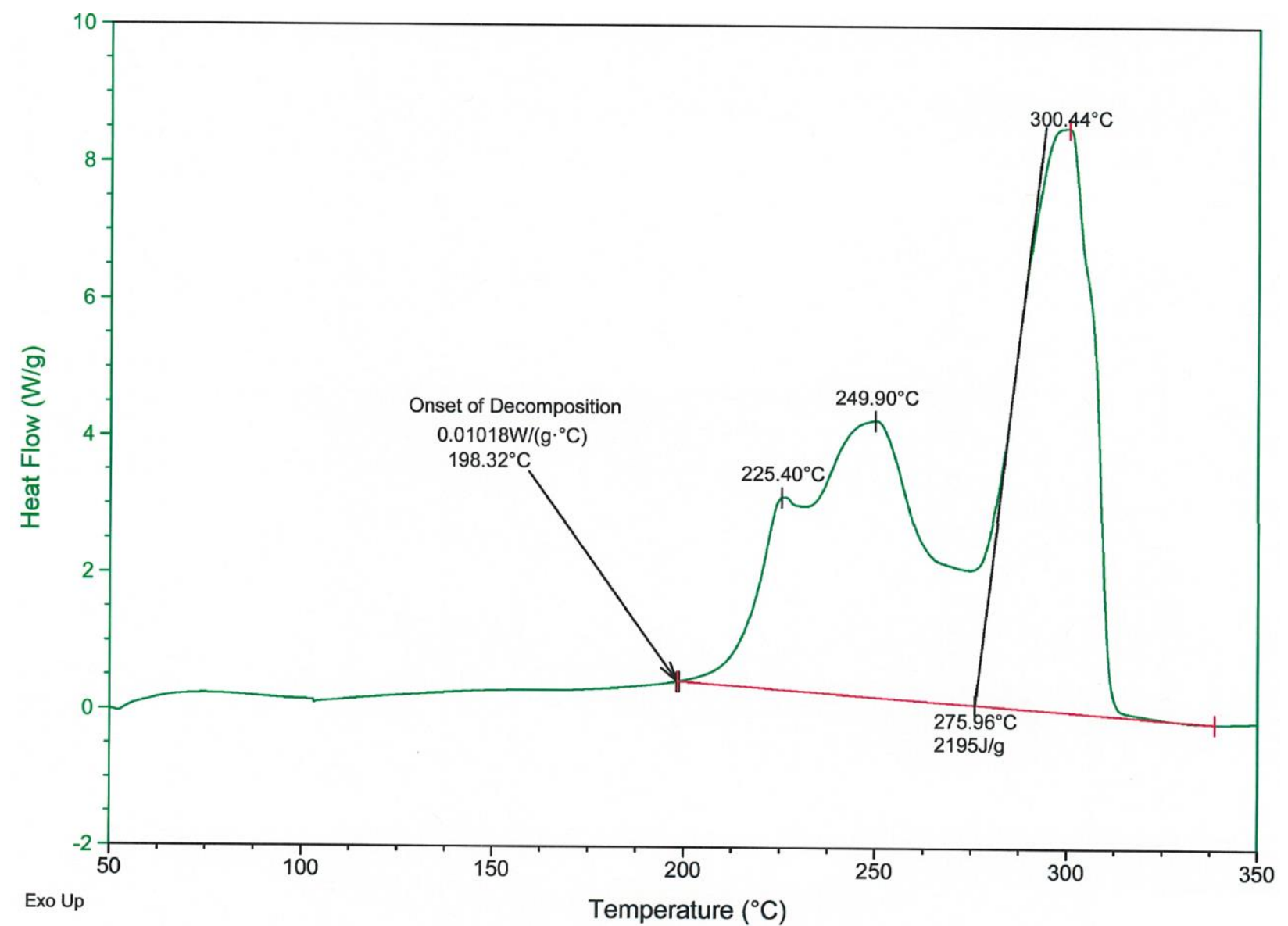

Figure S6. DSC graph of $\left[\mathrm{Fe}(\mathrm{Htrz})_{3}\right]_{n}\left[\mathrm{ClO}_{4}\right]_{2 n}$ (1) (exotherm up). 


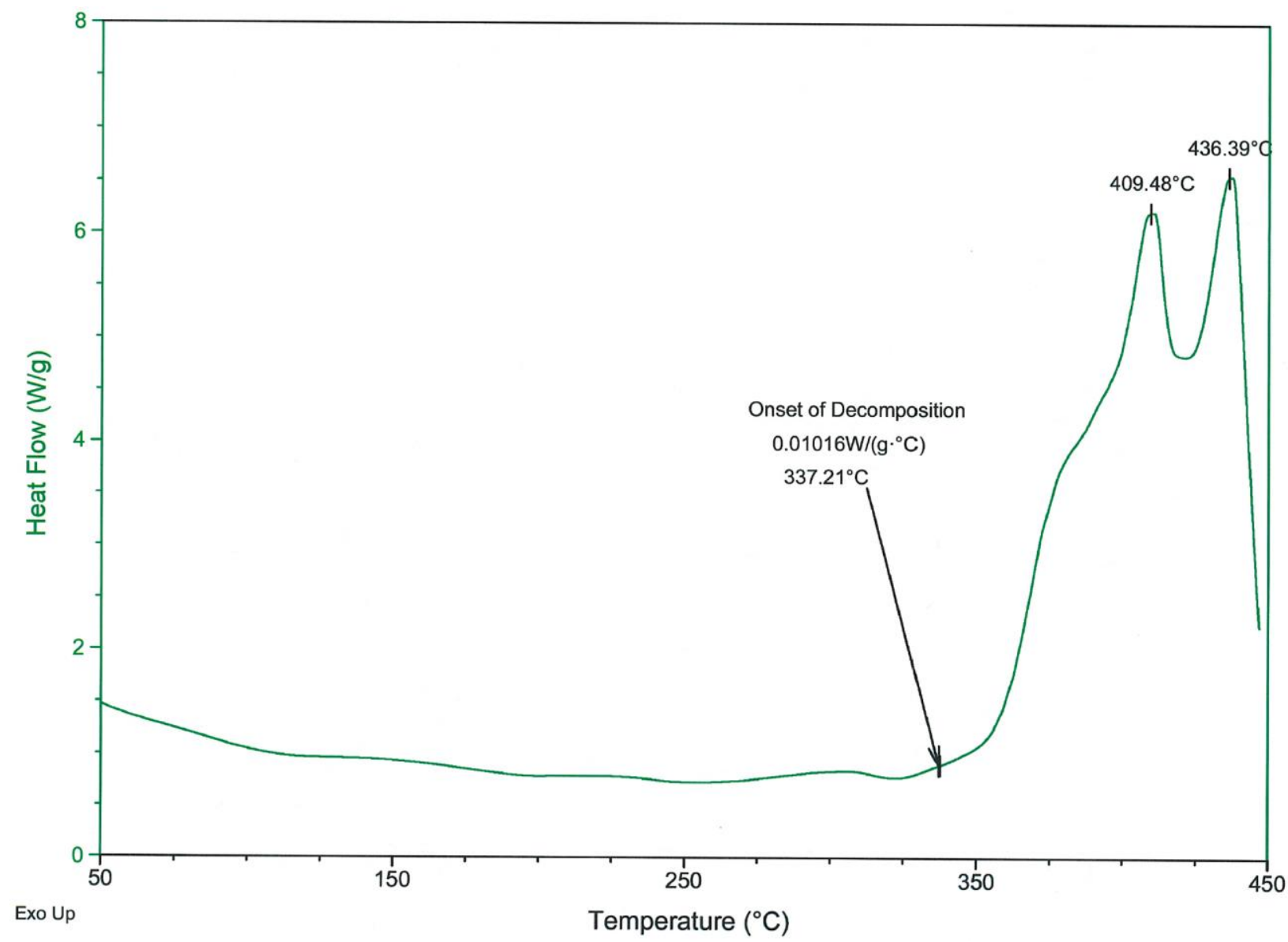

Figure S7. DSC graph of $\left[\mathrm{Ni}(\mathrm{Htrz})_{3}\right]_{n}\left[\mathrm{ClO}_{4}\right]_{2 n}$ (2) (exotherm up). 


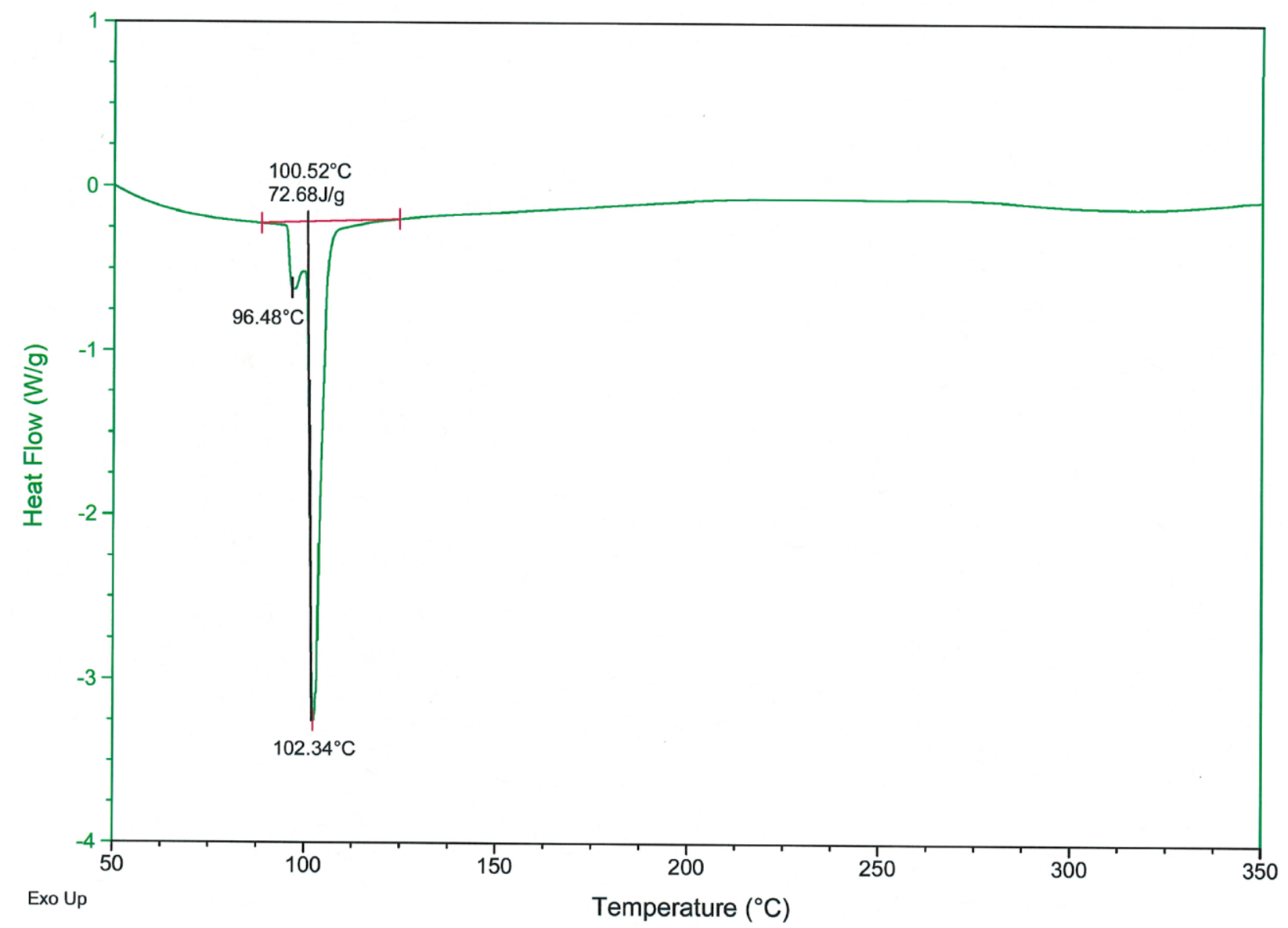

Figure S8. DSC graph of $\left[\mathrm{Fe}(\mathrm{Htrz})_{2}(\operatorname{trz})\right]_{n}\left[\mathrm{BF}_{4}\right]_{n}(3)$ (exotherm up). 


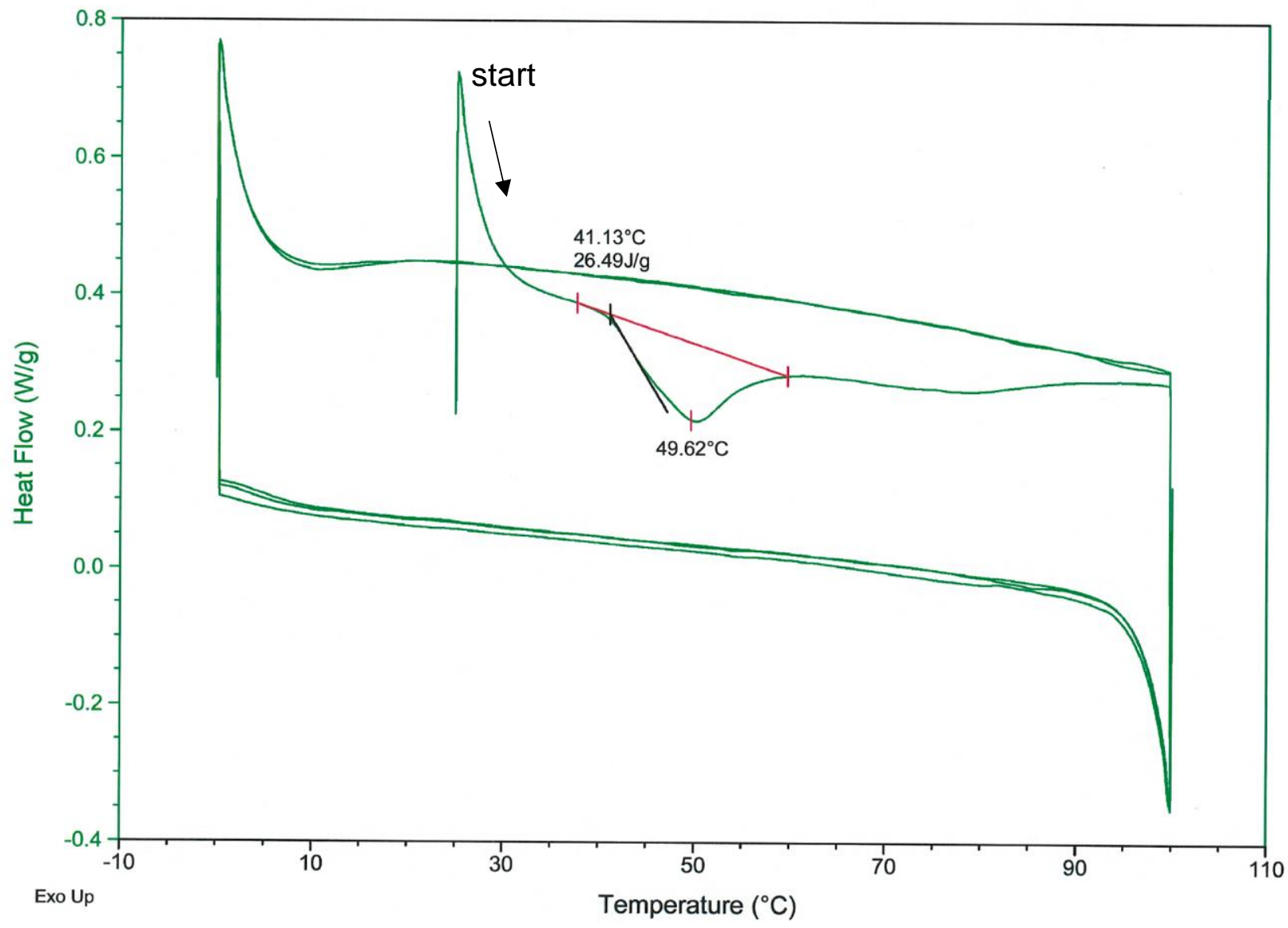

Figure S9. Cyclic DSC graph of $\left[\mathrm{Fe}(\mathrm{Htrz})_{3}\right]_{n}\left[\mathrm{ClO}_{4}\right]_{2 n}$ (1) (exotherm up). 


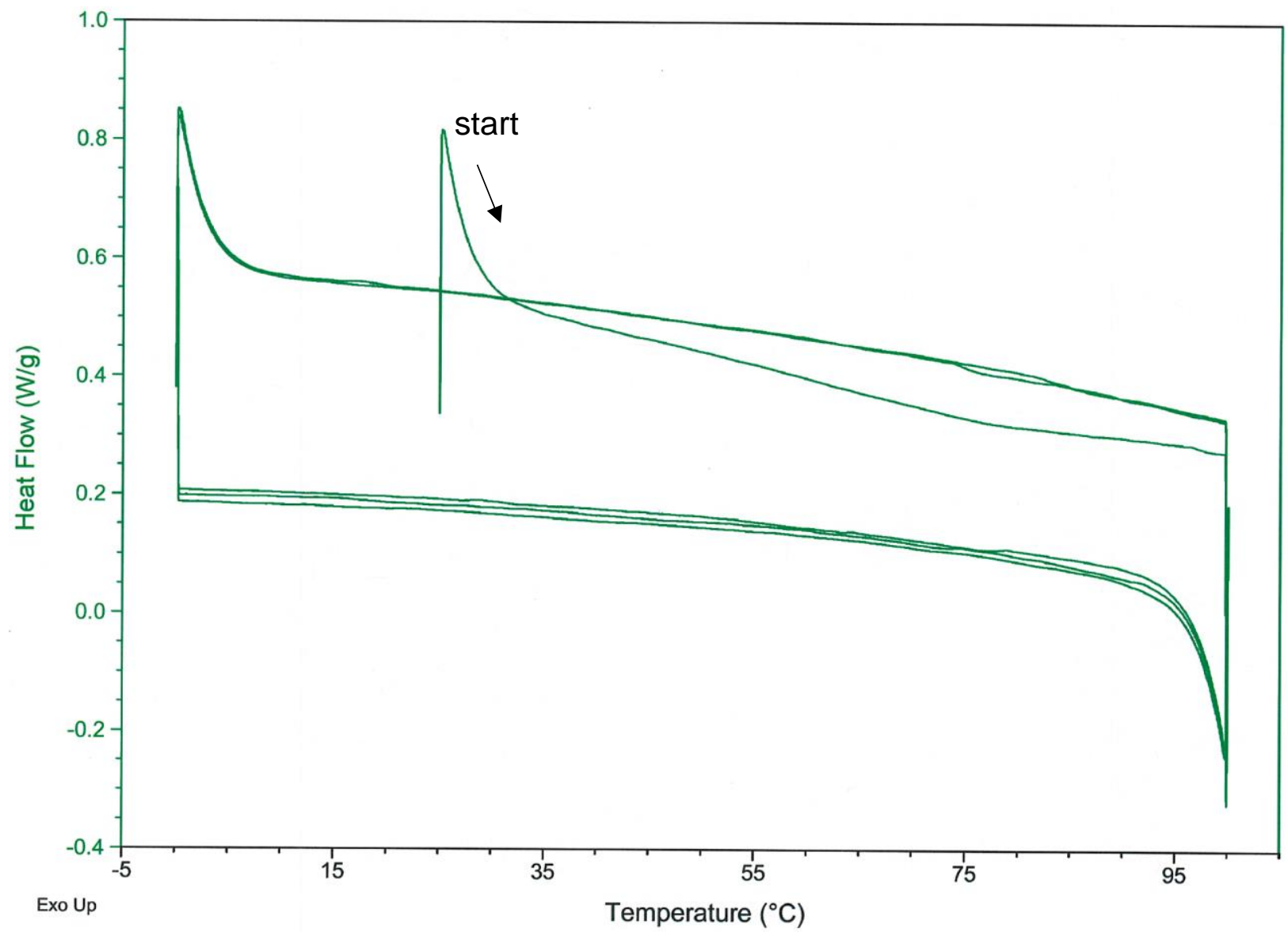

Figure S10. Cyclic DSC graph of $\left[\mathrm{Ni}(\mathrm{Htrz})_{3}\right]_{n}\left[\mathrm{ClO}_{4}\right]_{2 n}$ (2) (exotherm up). 


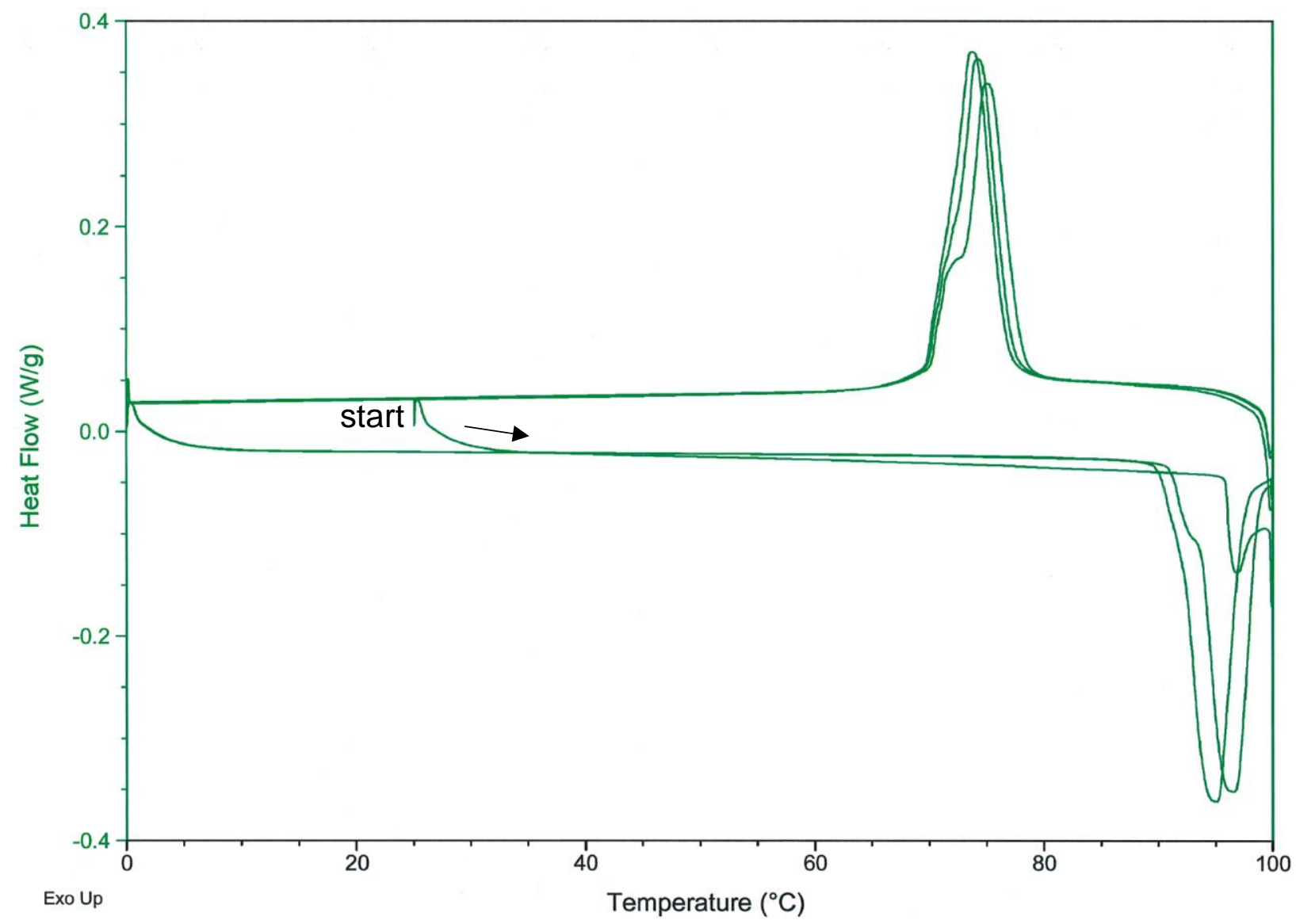

Figure S11. Cyclic DSC graph of $\left[\mathrm{Fe}(\mathrm{Htrz})_{2}(\operatorname{trz})\right]_{n}\left[\mathrm{BF}_{4}\right]_{n}(\mathbf{3})$ (exotherm up). 


\section{TGA Data}

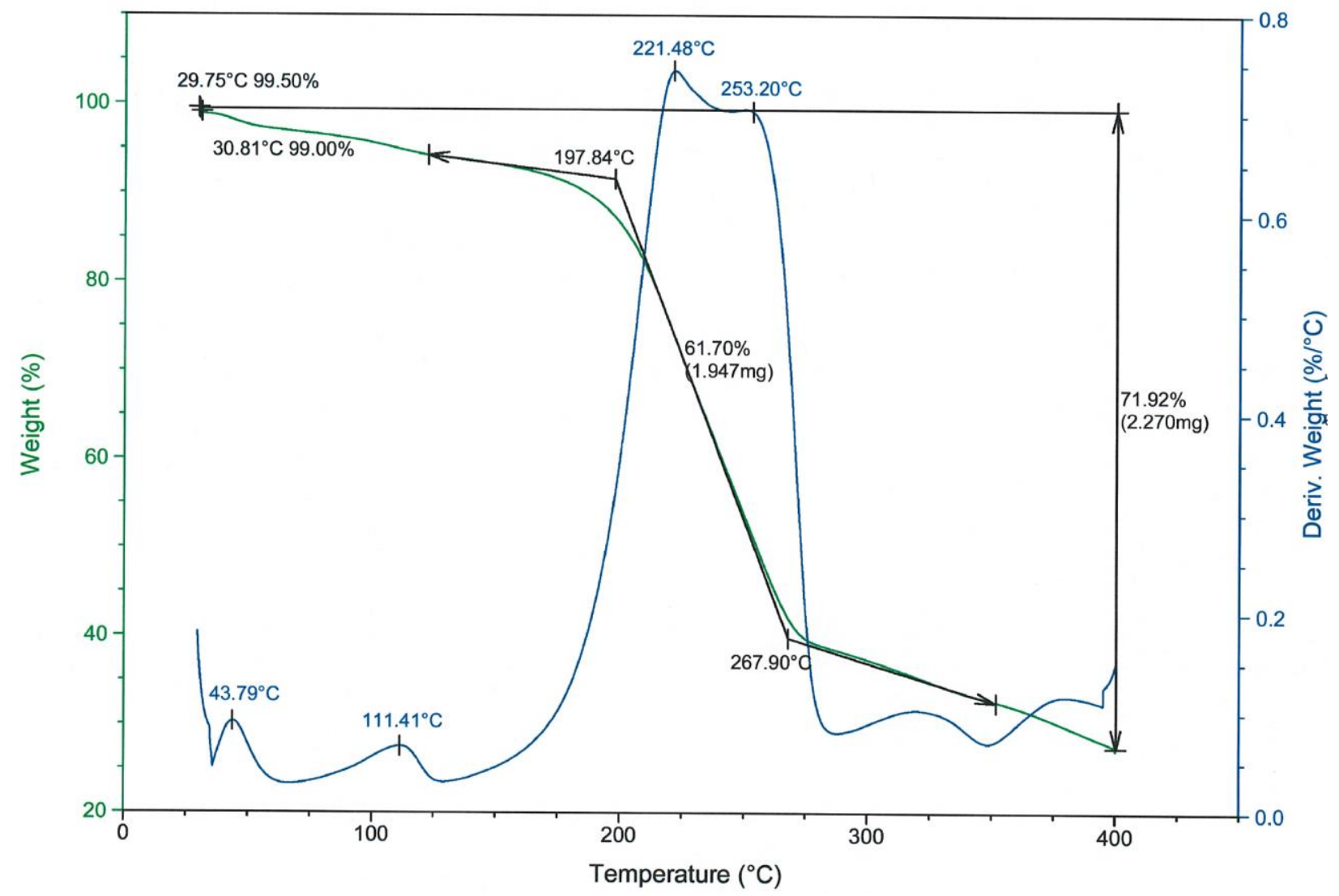

Figure S12. TGA graph of $\left[\mathrm{Fe}(\mathrm{Htrz})_{3}\right]_{n}\left[\mathrm{ClO}_{4}\right]_{2 n}$ (1) from 30 to $400{ }^{\circ} \mathrm{C}$ (3.4560 mg sample). 


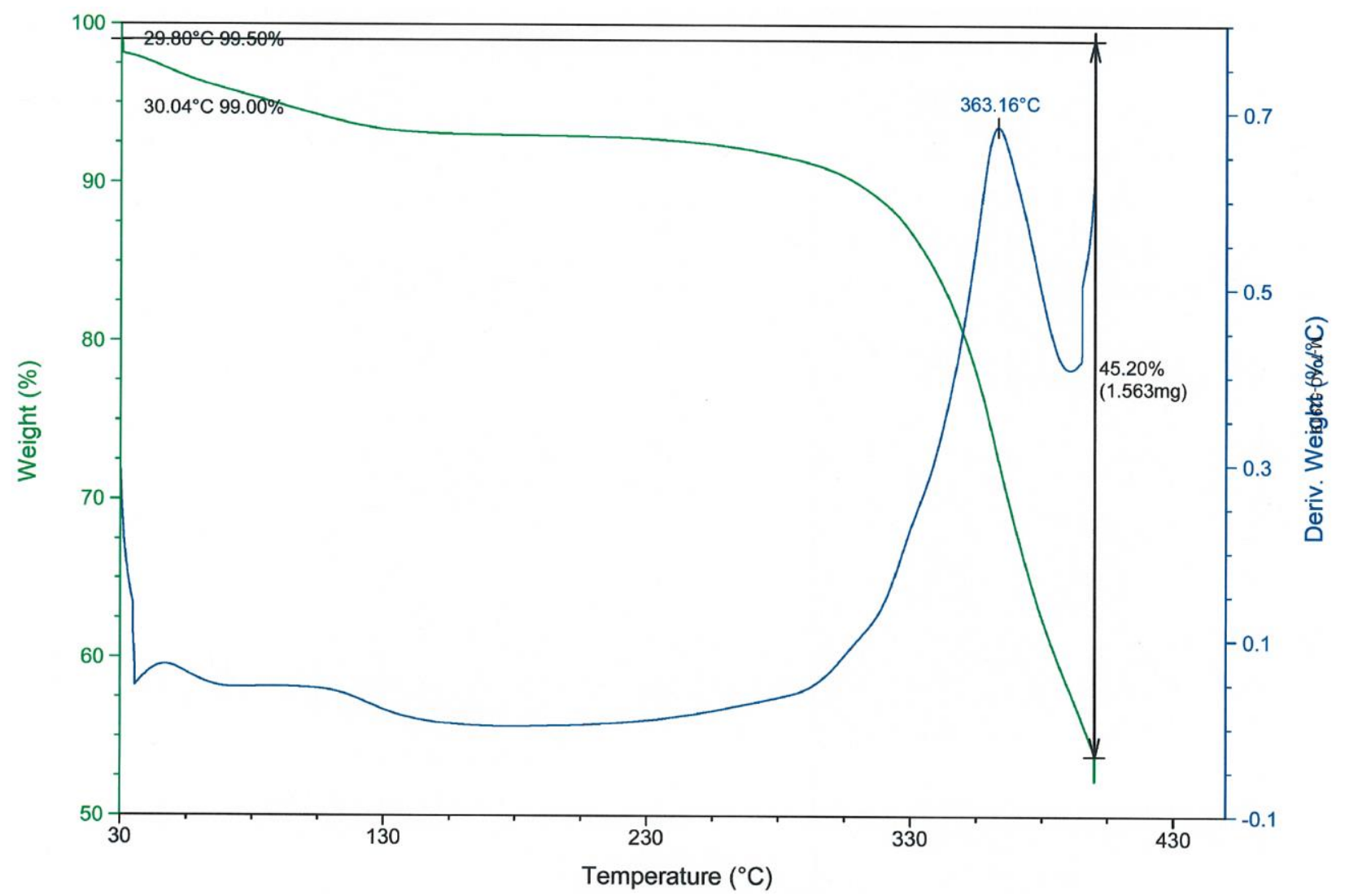

Figure S13. TGA graph of $\left[\mathrm{Ni}(\mathrm{Htrz})_{3}\right]_{n}\left[\mathrm{ClO}_{4}\right]_{2 n}$ (2) from 30 to $400{ }^{\circ} \mathrm{C}(3.4570 \mathrm{mg}$ sample). 


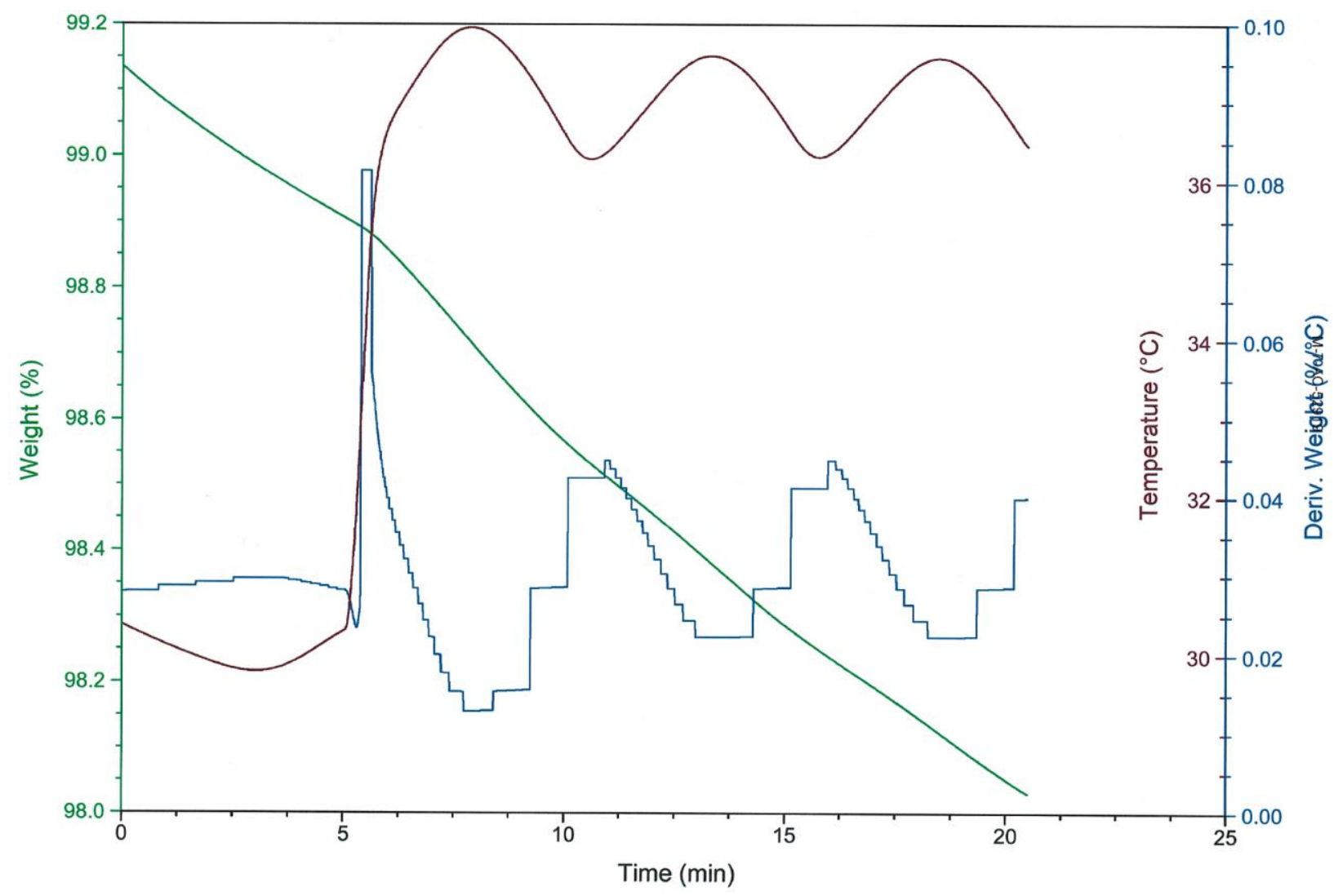

Figure S14. TGA graph of $\left[\mathrm{Fe}(\mathrm{Htrz})_{3}\right]_{n}\left[\mathrm{ClO}_{4}\right]_{2 n}$ (1) equilibrated at $30{ }^{\circ} \mathrm{C}$ for 5 min then ramped to $37{ }^{\circ} \mathrm{C}$ for $15 \mathrm{~min}$ (40.0040 $\mathrm{mg}$ sample). 


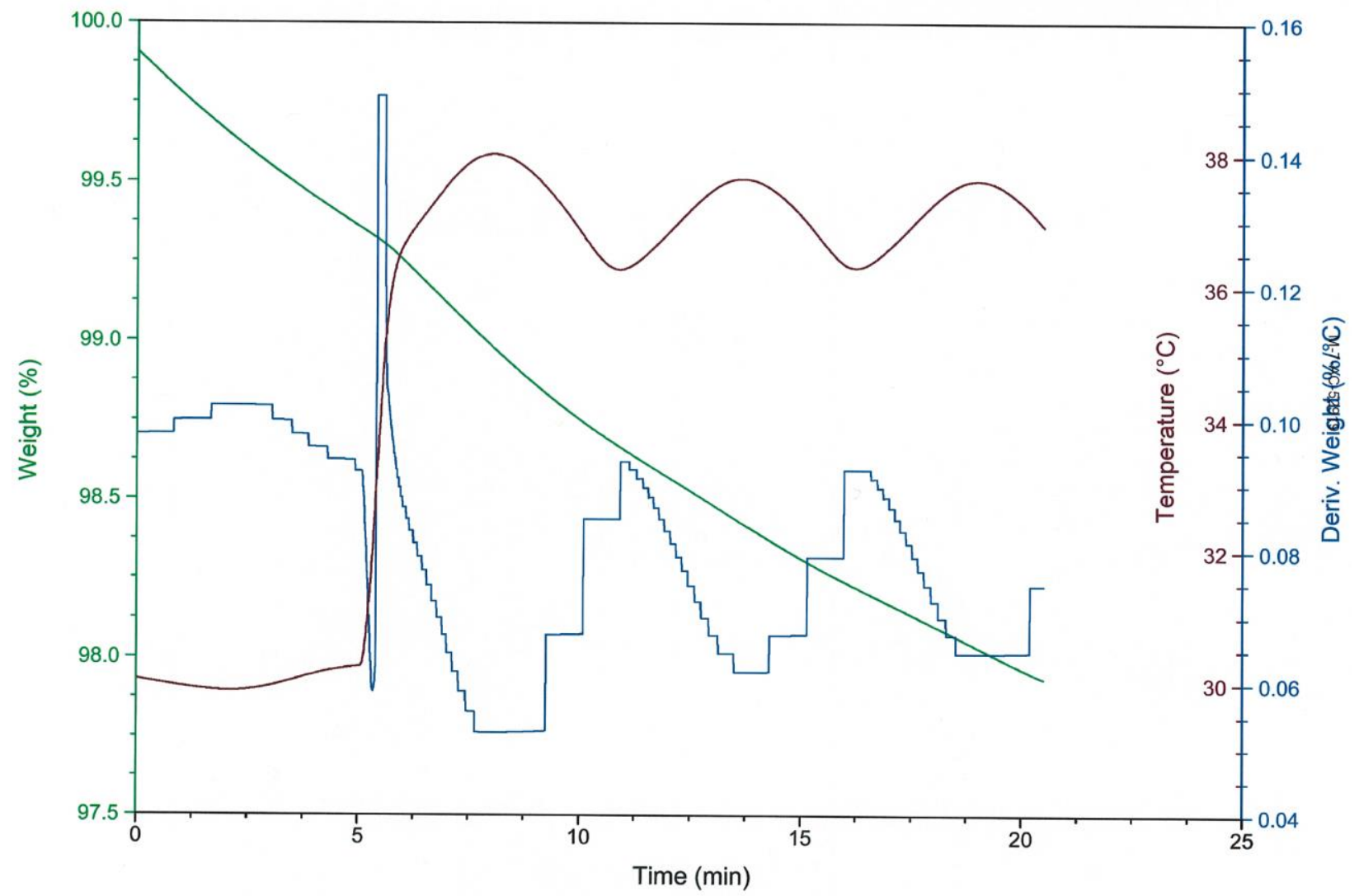

Figure S15. TGA graph of $\left[\mathrm{Ni}(\mathrm{Htrz})_{3}\right]_{n}\left[\mathrm{ClO}_{4}\right]_{2 n}$ (2) equilibrated at $30{ }^{\circ} \mathrm{C}$ for 5 min then ramped to $37{ }^{\circ} \mathrm{C}$ for $15 \mathrm{~min}(40.3450 \mathrm{mg}$ sample). 


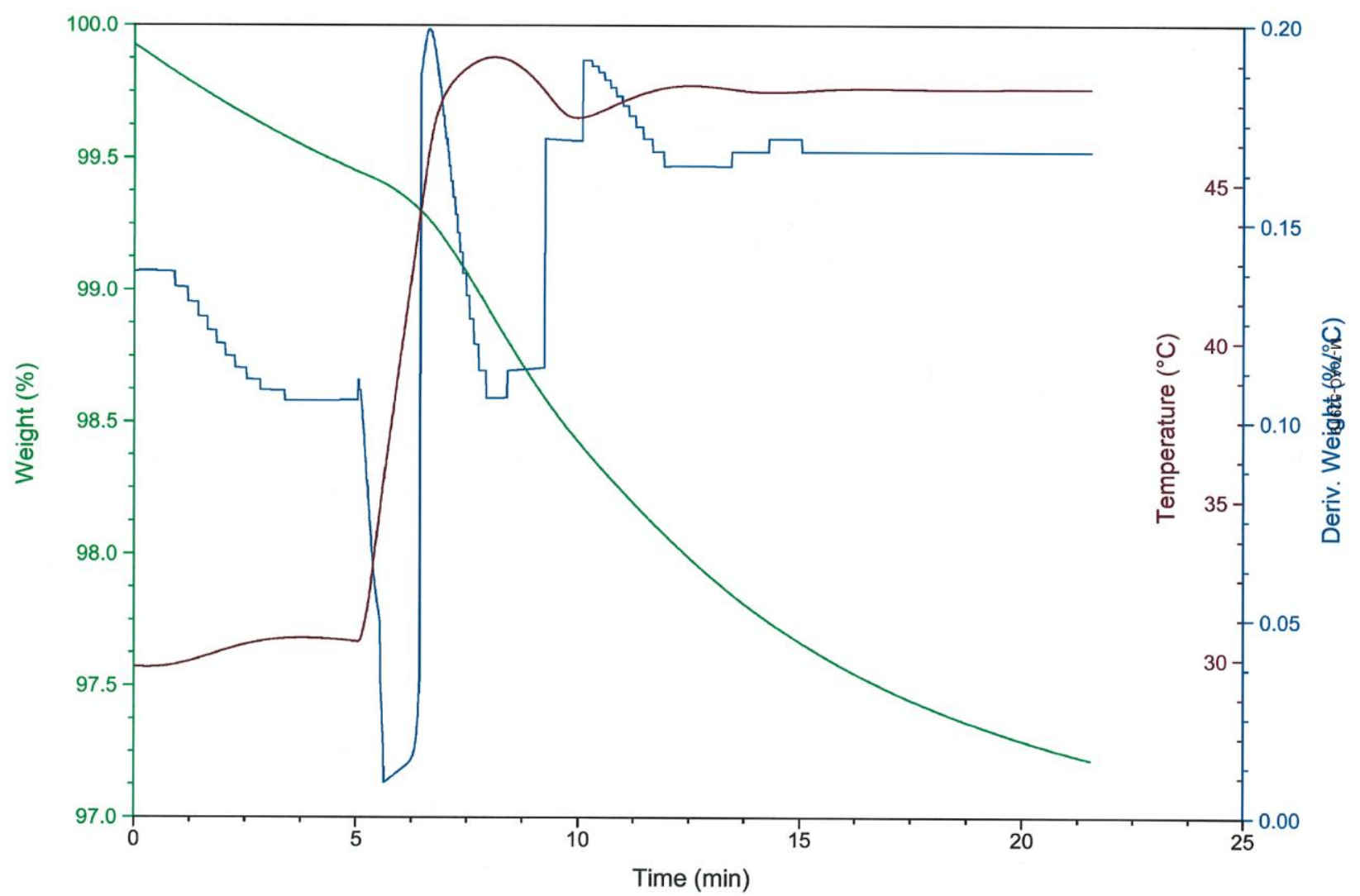

Figure S16. TGA graph of $\left[\mathrm{Fe}(\mathrm{Htrz})_{3}\right]_{n}\left[\mathrm{ClO}_{4}\right]_{2 n}$ (1) equilibrated at $30{ }^{\circ} \mathrm{C}$ for $5 \mathrm{~min}$ then ramped to $48{ }^{\circ} \mathrm{C}$ for $15 \mathrm{~min}(39.9160 \mathrm{mg}$ sample). 


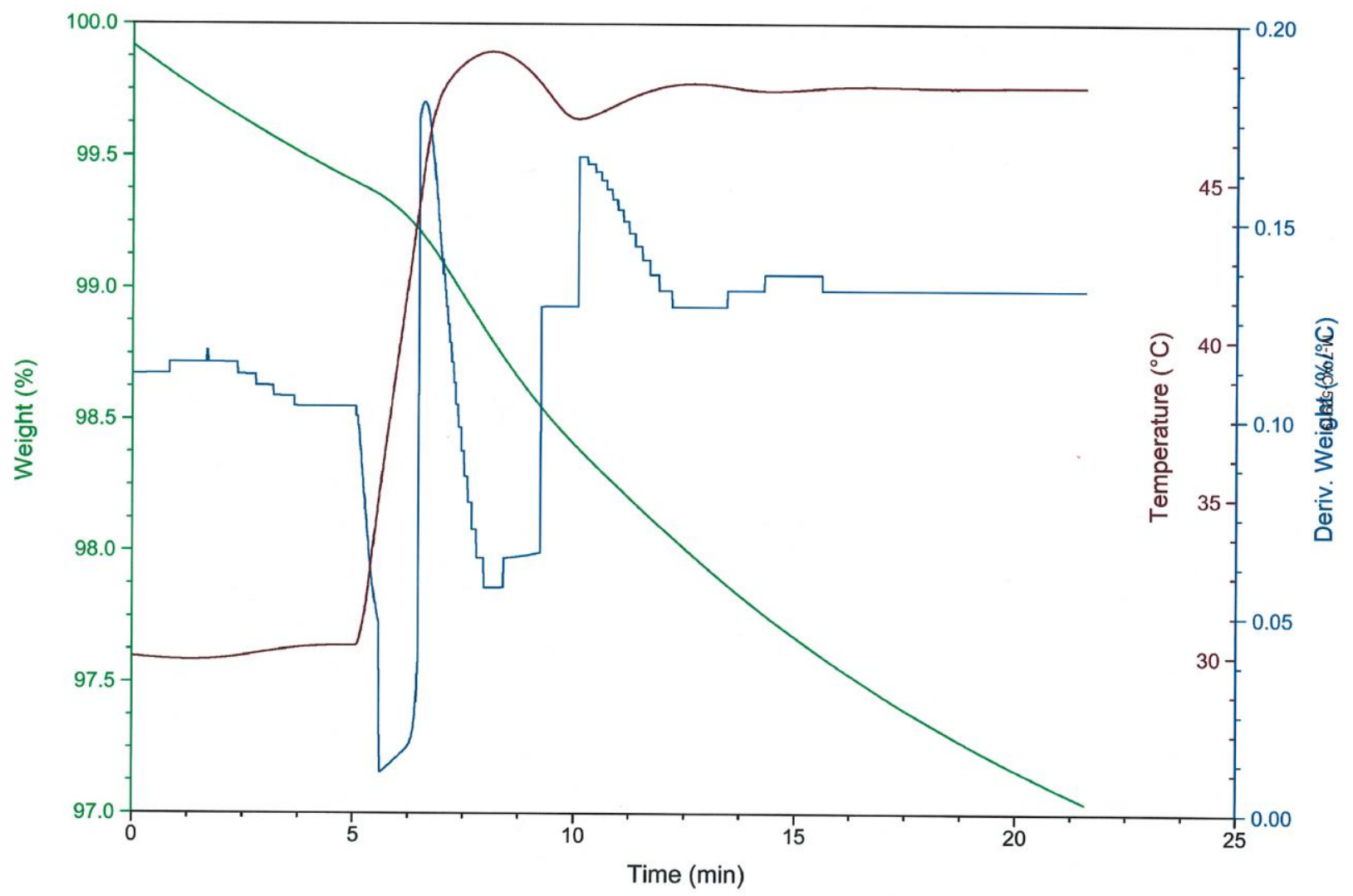

Figure S17. TGA graph of $\left[\mathrm{Ni}(\mathrm{Htrz})_{3}\right]_{n}\left[\mathrm{ClO}_{4}\right]_{2 n}$ (2) equilibrated at $30{ }^{\circ} \mathrm{C}$ for $5 \mathrm{~min}$ then ramped to $48{ }^{\circ} \mathrm{C}$ for $15 \mathrm{~min}(40.7210 \mathrm{mg}$ sample). 


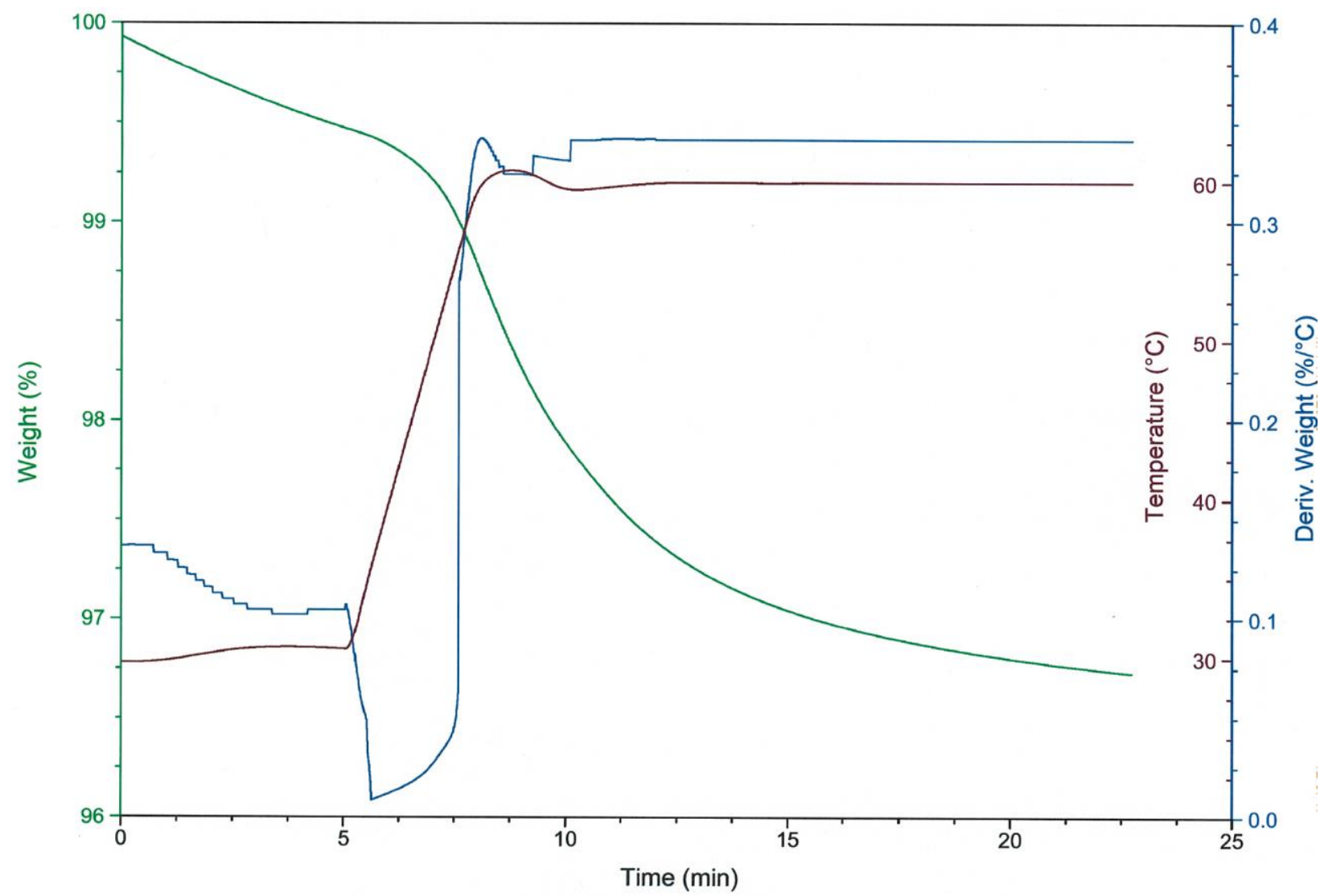

Figure S18. TGA graph of $\left[\mathrm{Fe}(\mathrm{Htrz})_{3}\right]_{n}\left[\mathrm{ClO}_{4}\right]_{2 n}$ (1) equilibrated at $30{ }^{\circ} \mathrm{C}$ for 5 min then ramped to $60{ }^{\circ} \mathrm{C}$ for $15 \mathrm{~min}$ (40.0990 $\mathrm{mg}$ sample). 


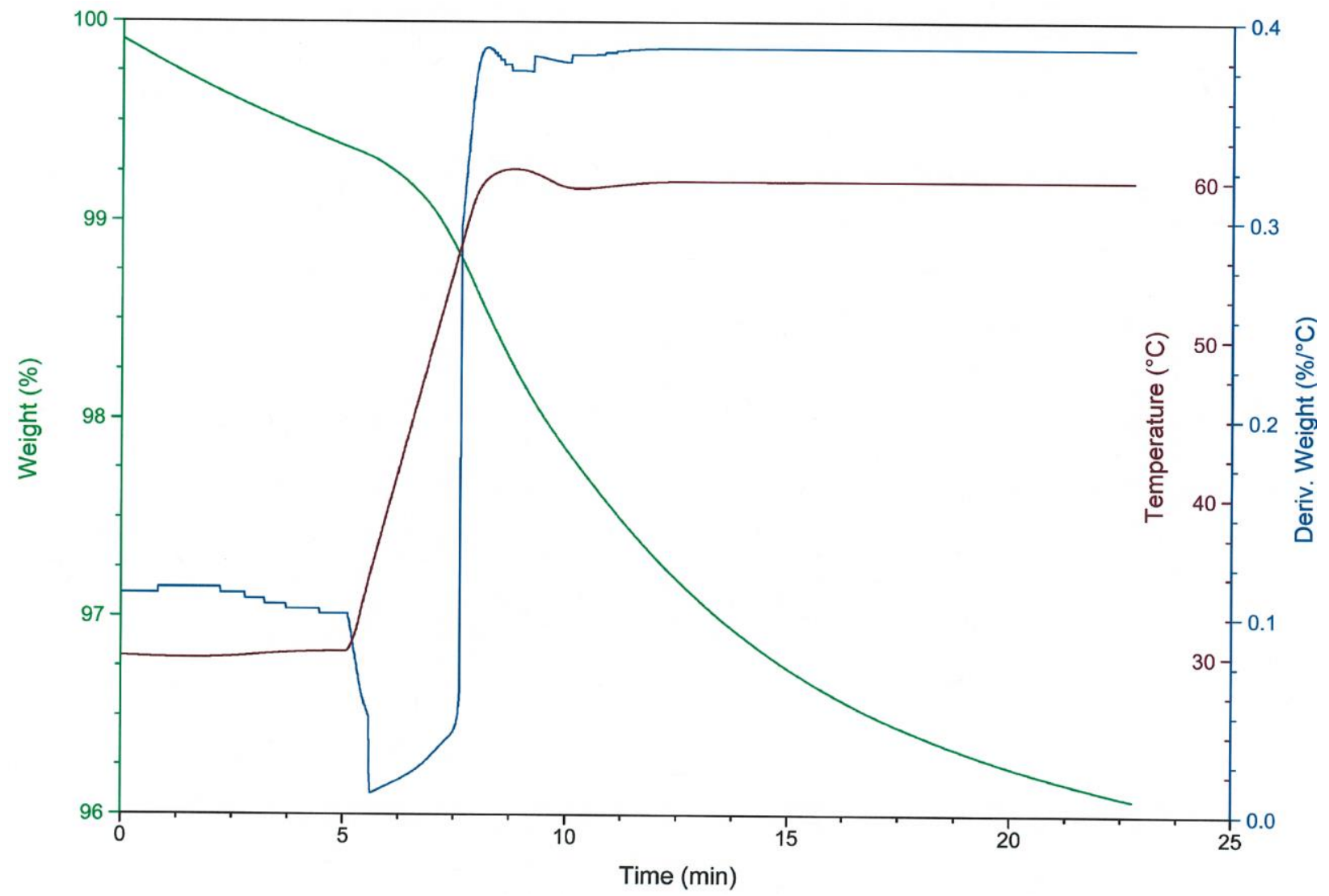

Figure S19. TGA graph of $\left[\mathrm{Ni}(\mathrm{Htrz})_{3}\right]_{n}\left[\mathrm{ClO}_{4}\right]_{2 n}$ (2) equilibrated at $30{ }^{\circ} \mathrm{C}$ for 5 min then ramped to $60{ }^{\circ} \mathrm{C}$ for $15 \mathrm{~min}$ (40.4980 $\mathrm{mg}$ sample). 


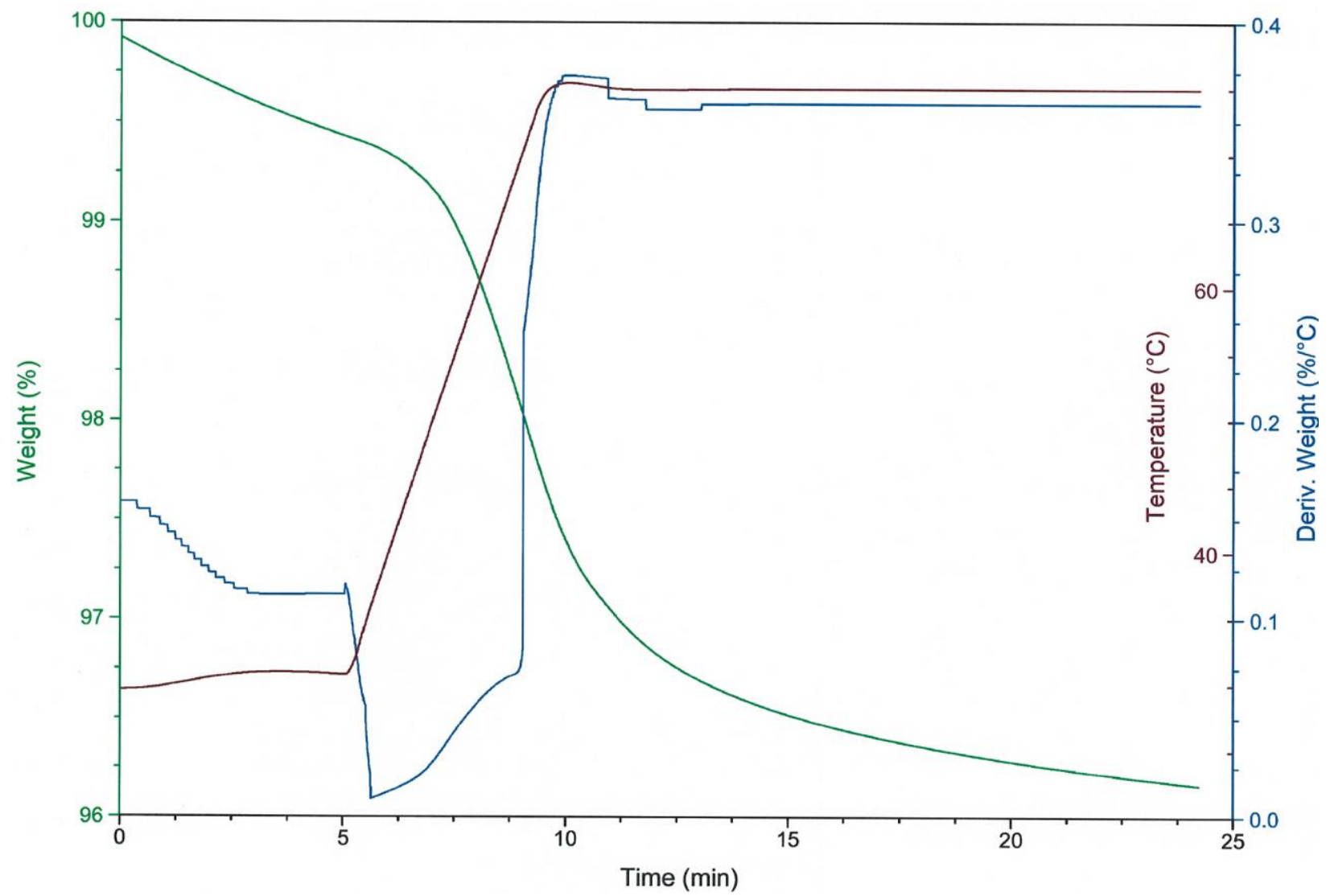

Figure S20. TGA graph of $\left[\mathrm{Fe}(\mathrm{Htrz})_{3}\right]_{n}\left[\mathrm{ClO}_{4}\right]_{2 n}$ (1) equilibrated at $30{ }^{\circ} \mathrm{C}$ for 5 min then ramped to $75^{\circ} \mathrm{C}$ for $15 \mathrm{~min}$ (39.9120 $\mathrm{mg}$ sample). 


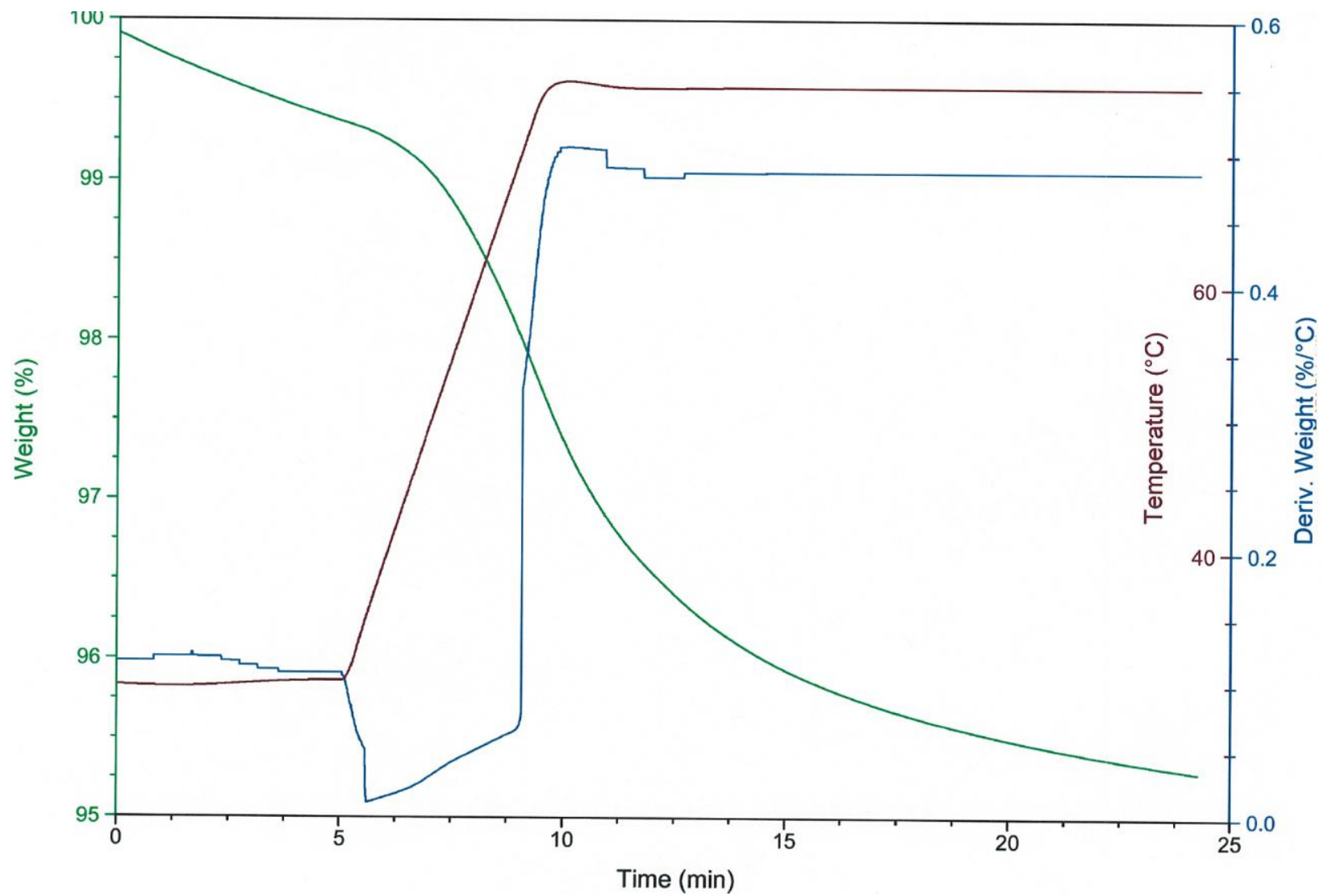

Figure S21. TGA graph of $\left[\mathrm{Ni}(\mathrm{Htrz})_{3}\right]_{n}\left[\mathrm{ClO}_{4}\right]_{2 n}$ (2) equilibrated at $30{ }^{\circ} \mathrm{C}$ for 5 min then ramped to $75{ }^{\circ} \mathrm{C}$ for $15 \mathrm{~min}(40.5620 \mathrm{mg}$ sample). 


\section{Impact Sensitivity Test Data}

Impact sensitivity data was collected with a go / no-go determination threshold of $117 \mathrm{~dB}$, using the Neyer D-Optimal method to determine at which drop heights to collect data for the most efficient test. On examination of the data (Figure S22 and Tables S1-10), we determined that a go / no-go determination threshold of $105 \mathrm{~dB}$ was more appropriate for our compounds and $\mathrm{H}_{50}$ drop heights were calculated using the lower threshold. At the initial $117 \mathrm{~dB}$ threshold, there is always a clear clustering in sound intensity that facilitates the distinction between go and no-go events in compound 2 (Figure S22f-j). According to this clustering, there is only one drop (48 ${ }^{\circ} \mathrm{C}, 27 \mathrm{~cm}, 115.35 \mathrm{~cm}$ ) that falls below the $117 \mathrm{~dB}$ threshold but appears to be a "go." In contrast, there are several drops on 1 that are classified as "no-goes" at the $117 \mathrm{~dB}$ threshold, even though some of the material appeared to be consumed by visual inspection. This noisier data for $\mathbf{1}$ is due to it being less powerful and thus producing less sound intensity than $\mathbf{2}$ upon reaction. The lower sound intensity cluster ("no goes") in the data for $\mathbf{2}$ is $97.05-101.85 \mathrm{~dB}$. Since both $\mathbf{1}$ and $\mathbf{2}$ are fine powders that should yield comparable sound intensities for "nogoes," a go/no-go determination threshold of $105 \mathrm{~dB}$ was selected for calculation of $\mathrm{H}_{50}$ drop heights. 

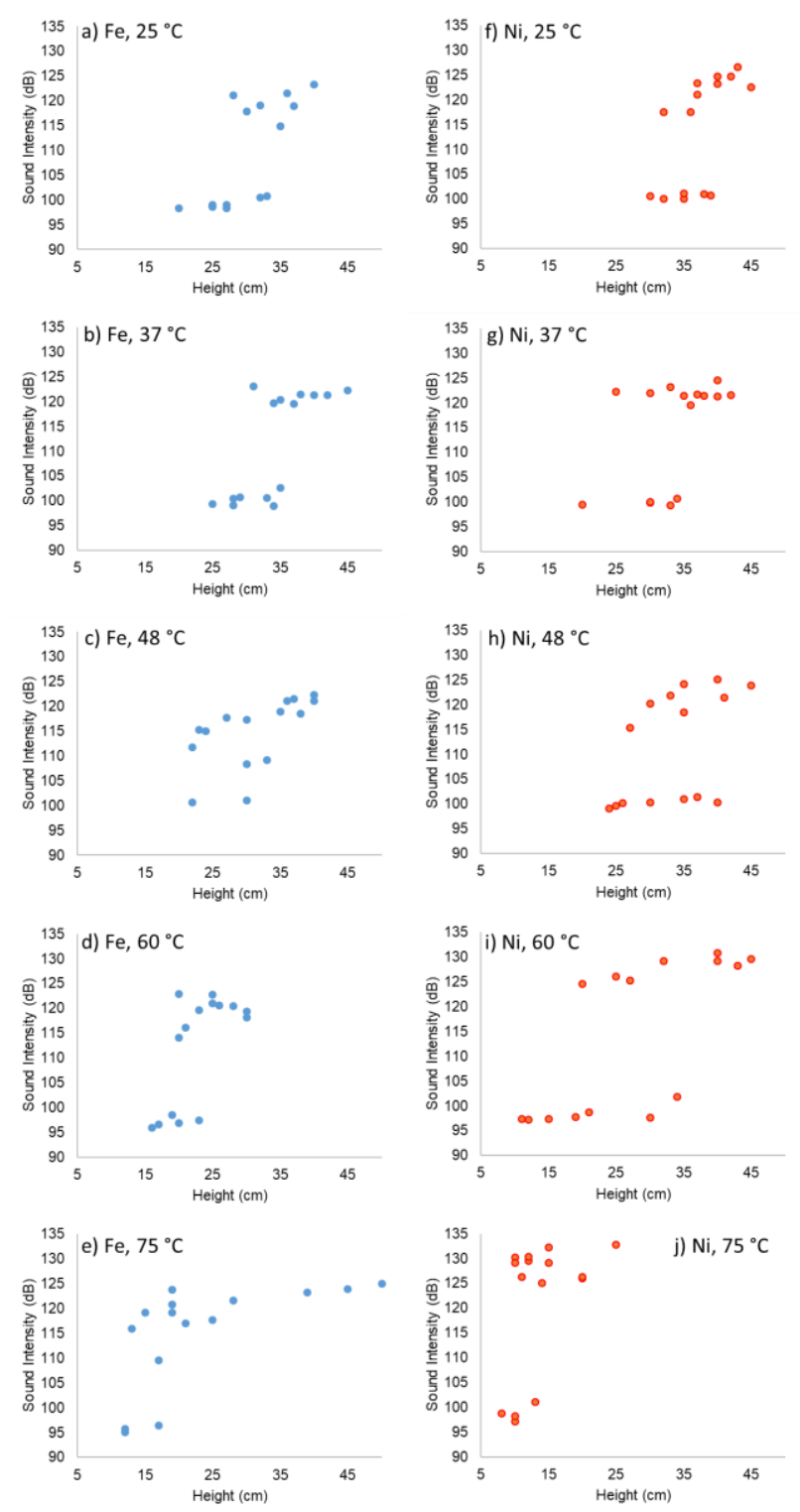

Figure S22. Drop hammer test results showing the drop height vs. average maximum sound intensity. Each graph is a set of 15 drops, at the same temperature, from which the $\mathrm{H}_{50}$ is calculated. Every data point represents one drop. Graphs a-e) are the results for $\left[\mathrm{Fe}(\mathrm{Htrz})_{3}\right]_{n}\left[\mathrm{ClO}_{4}\right]_{2 n}(\mathbf{1})$ and are labelled with $\mathrm{Fe}$ and the respective test temperatures. Graphs $\mathrm{f}-\mathrm{j}$ ) are the results for $\left[\mathrm{Ni}(\mathrm{Htrz})_{3}\right]_{n}\left[\mathrm{ClO}_{4}\right]_{2 n}(\mathbf{2})$ and are labelled with $\mathrm{Ni}$ and the respective test temperatures. 
Table S1. Impact sensitivity test acoustic data on $\left[\mathrm{Fe}(\mathrm{Htrz})_{3}\right]_{n}\left[\mathrm{ClO}_{4}\right]_{2 n}(\mathbf{1})$ at $25^{\circ} \mathrm{C}$.

\begin{tabular}{cccc}
\hline Drop Height $(\mathrm{cm})$ & Microphone 1 $(\mathrm{dB})$ & Microphone 2 $(\mathrm{dB})$ & Avg. of 1 and 2 $(\mathrm{dB})$ \\
\hline 40 & 121.7 & 124.8 & 123.25 \\
30 & 117.1 & 118.6 & 117.85 \\
20 & 97.6 & 99.1 & 98.35 \\
25 & 97.6 & 99.6 & 98.6 \\
35 & 113.3 & 116.4 & 114.85 \\
27 & 98.2 & 99.7 & 98.95 \\
32 & 117.2 & 120.8 & 119 \\
28 & 119.8 & 122.5 & 121.15 \\
25 & 98.1 & 99.5 & 98.8 \\
32 & 99.6 & 101.3 & 100.45 \\
37 & 115.4 & 122.5 & 118.95 \\
33 & 98.8 & 102.8 & 100.8 \\
25 & 98.4 & 99.5 & 98.95 \\
36 & 120.6 & 122.3 & 121.45 \\
27 & 96.5 & 100.2 & 98.35 \\
\hline
\end{tabular}

Table S2. Impact sensitivity test acoustic data on $\left[\mathrm{Fe}(\mathrm{Htrz})_{3}\right]_{n}\left[\mathrm{ClO}_{4}\right]_{2 n}(\mathbf{1})$ at $37{ }^{\circ} \mathrm{C}$.

\begin{tabular}{cccc}
\hline Drop Height $(\mathrm{cm})$ & Microphone 1 $(\mathrm{dB})$ & Microphone 2 $(\mathrm{dB})$ & Avg. of 1 and 2 $(\mathrm{dB})$ \\
\hline 40 & 119.1 & 123.6 & 121.35 \\
34 & 117.1 & 122.3 & 119.7 \\
28 & 99.3 & 101.6 & 100.45 \\
31 & 122.6 & 123.5 & 123.05 \\
25 & 98.4 & 100.2 & 99.3 \\
33 & 99.6 & 101.5 & 100.55 \\
42 & 120.1 & 122.4 & 121.25 \\
37 & 118.7 & 120.3 & 119.5 \\
34 & 98.2 & 99.7 & 98.95 \\
45 & 121.4 & 123.2 & 122.3 \\
35 & 119.5 & 121.2 & 120.35 \\
28 & 98.8 & 99.3 & 99.05 \\
38 & 120.4 & 122.5 & 121.45 \\
29 & 99.6 & 101.8 & 100.7 \\
35 & 101.9 & 103.2 & 102.55 \\
\hline
\end{tabular}


Table S3. Impact sensitivity test acoustic data on $\left[\mathrm{Fe}(\mathrm{Htrz})_{3}\right]_{n}\left[\mathrm{ClO}_{4}\right]_{2 n}(\mathbf{1})$ at $48{ }^{\circ} \mathrm{C}$.

\begin{tabular}{cccc}
\hline Drop Height $(\mathrm{cm})$ & Microphone 1 $(\mathrm{dB})$ & Microphone 2 $(\mathrm{dB})$ & Avg. of 1 and 2 $(\mathrm{dB})$ \\
\hline 30 & 115.2 & 119.3 & 117.25 \\
24 & 114.4 & 115.7 & 115.05 \\
35 & 118.3 & 119.6 & 118.95 \\
27 & 116.4 & 119.1 & 117.75 \\
22 & 109.5 & 114.1 & 111.8 \\
30 & 107.2 & 109.4 & 108.3 \\
40 & 119.4 & 125.2 & 122.3 \\
33 & 108.3 & 110.1 & 109.2 \\
38 & 116.7 & 120.3 & 118.5 \\
22 & 99.8 & 101.5 & 100.65 \\
37 & 119.1 & 123.9 & 121.5 \\
23 & 114.4 & 116 & 115.2 \\
36 & 118.9 & 123.4 & 121.15 \\
30 & 100.1 & 102 & 101.05 \\
40 & 118.4 & 123.9 & 121.15 \\
\hline
\end{tabular}

Table S4. Impact sensitivity test acoustic data on $\left[\mathrm{Fe}(\mathrm{Htrz})_{3}\right]_{n}\left[\mathrm{ClO}_{4}\right]_{2 n}(\mathbf{1})$ at $60{ }^{\circ} \mathrm{C}$.

\begin{tabular}{cccc}
\hline Drop Height $(\mathrm{cm})$ & Microphone 1 $(\mathrm{dB})$ & Microphone 2 $(\mathrm{dB})$ & Avg. of 1 and 2 $(\mathrm{dB})$ \\
\hline 20 & 112 & 116.1 & 114.05 \\
30 & 117.7 & 121 & 119.35 \\
25 & 120.9 & 121 & 120.95 \\
20 & 121.1 & 124.6 & 122.85 \\
16 & 95.3 & 96.4 & 95.85 \\
23 & 116.2 & 123 & 119.6 \\
23 & 95.1 & 99.7 & 97.4 \\
28 & 118.1 & 122.8 & 120.45 \\
17 & 94.5 & 98.6 & 96.55 \\
30 & 116 & 120.2 & 118.1 \\
21 & 113.7 & 118.4 & 116.05 \\
26 & 118.1 & 122.9 & 120.5 \\
19 & 95.4 & 101.5 & 98.45 \\
25 & 121.1 & 124.5 & 122.8 \\
20 & 95.1 & 98.7 & 96.9 \\
\hline
\end{tabular}


Table S5. Impact sensitivity test acoustic data on $\left[\mathrm{Fe}(\mathrm{Htrz})_{3}\right]_{n}\left[\mathrm{ClO}_{4}\right]_{2 n}(\mathbf{1})$ at $75^{\circ} \mathrm{C}$.

\begin{tabular}{cccc}
\hline Drop Height $(\mathrm{cm})$ & Microphone 1 $(\mathrm{dB})$ & Microphone 2 $(\mathrm{dB})$ & Avg. of 1 and 2 $(\mathrm{dB})$ \\
\hline 50 & 124.1 & 125.9 & 125 \\
45 & 123 & 124.9 & 123.95 \\
39 & 122 & 124.6 & 123.3 \\
28 & 120.5 & 122.8 & 121.65 \\
19 & 119.5 & 122 & 120.75 \\
12 & 94.2 & 97.3 & 95.75 \\
15 & 118.2 & 120.1 & 119.15 \\
12 & 93.5 & 96.5 & 95 \\
13 & 114 & 117.9 & 115.95 \\
17 & 107.7 & 111.3 & 109.5 \\
21 & 116.7 & 117.4 & 117.05 \\
19 & 122.7 & 124.8 & 123.75 \\
17 & 94.7 & 98 & 96.35 \\
25 & 115.3 & 120.2 & 117.75 \\
19 & 118 & 120.3 & 119.15 \\
\hline
\end{tabular}

Table S6. Impact sensitivity test acoustic data on $\left[\mathrm{Ni}(\mathrm{Htrz})_{3}\right]_{n}\left[\mathrm{ClO}_{4}\right]_{2 n}(2)$ at $25^{\circ} \mathrm{C}$.

\begin{tabular}{cccc}
\hline Drop Height $(\mathrm{cm})$ & Microphone 1 $(\mathrm{dB})$ & Microphone 2 $(\mathrm{dB})$ & Avg. of 1 and 2 $(\mathrm{dB})$ \\
\hline 40 & 121.6 & 124.8 & 123.2 \\
35 & 99.3 & 100.8 & 100.05 \\
45 & 121.3 & 123.7 & 122.5 \\
37 & 118.8 & 123.2 & 121 \\
32 & 99.3 & 100.8 & 100.05 \\
35 & 99.6 & 102.6 & 101.1 \\
38 & 100.7 & 101.3 & 101 \\
42 & 123.2 & 126.3 & 124.75 \\
39 & 99.6 & 101.7 & 100.65 \\
43 & 125.6 & 127.7 & 126.65 \\
40 & 123.2 & 126.1 & 124.65 \\
36 & 117.4 & 117.5 & 117.45 \\
32 & 115.9 & 119.1 & 117.5 \\
30 & 99.4 & 101.6 & 100.5 \\
37 & 121.8 & 124.8 & 123.3 \\
\hline
\end{tabular}


Table S7. Impact sensitivity test acoustic data on $\left[\mathrm{Ni}(\mathrm{Htrz})_{3}\right]_{n}\left[\mathrm{ClO}_{4}\right]_{2 n}(\mathbf{2})$ at $37^{\circ} \mathrm{C}$.

\begin{tabular}{cccc}
\hline Drop Height $(\mathrm{cm})$ & Microphone 1 $(\mathrm{dB})$ & Microphone 2 $(\mathrm{dB})$ & Avg. of 1 and 2 $(\mathrm{dB})$ \\
\hline 40 & 122.7 & 126.4 & 124.55 \\
35 & 119.4 & 123.4 & 121.4 \\
30 & 98.4 & 101.3 & 99.85 \\
33 & 120.8 & 125.5 & 123.15 \\
30 & 119.3 & 124.5 & 121.9 \\
25 & 119.8 & 124.6 & 122.2 \\
20 & 98.7 & 100.2 & 99.45 \\
37 & 119.6 & 123.7 & 121.65 \\
34 & 100.2 & 101.1 & 100.65 \\
42 & 120.7 & 122.4 & 121.55 \\
38 & 120.4 & 122.3 & 121.35 \\
30 & 99.3 & 100.8 & 100.05 \\
36 & 118.6 & 120.5 & 119.55 \\
33 & 98.2 & 100.3 & 99.25 \\
40 & 120.5 & 122.1 & 121.3 \\
\hline
\end{tabular}

Table S8. Impact sensitivity test acoustic data on $\left[\mathrm{Ni}(\mathrm{Htrz})_{3}\right]_{n}\left[\mathrm{ClO}_{4}\right]_{2 n}(2)$ at $48{ }^{\circ} \mathrm{C}$.

\begin{tabular}{cccc}
\hline Drop Height $(\mathrm{cm})$ & Microphone 1 $(\mathrm{dB})$ & Microphone 2 $(\mathrm{dB})$ & Avg. of 1 and 2 $(\mathrm{dB})$ \\
\hline 40 & 123.3 & 126.8 & 125.05 \\
35 & 99.7 & 102.3 & 101 \\
45 & 122.1 & 125.6 & 123.85 \\
35 & 117.3 & 119.6 & 118.45 \\
30 & 99.4 & 101.3 & 100.35 \\
37 & 100.6 & 102.1 & 101.35 \\
41 & 120.3 & 122.5 & 121.4 \\
33 & 120.2 & 123.5 & 121.85 \\
30 & 120.1 & 120.2 & 120.15 \\
25 & 98.1 & 101.1 & 99.6 \\
27 & 112.1 & 118.6 & 115.35 \\
35 & 122.1 & 126.2 & 124.15 \\
24 & 98 & 100.2 & 99.1 \\
26 & 98.9 & 101.5 & 100.2 \\
40 & 99.2 & 101.3 & 100.25 \\
\hline
\end{tabular}


Table S9. Impact sensitivity test acoustic data on $\left[\mathrm{Ni}(\mathrm{Htrz})_{3}\right]_{n}\left[\mathrm{ClO}_{4}\right]_{2 n}(2)$ at $60{ }^{\circ} \mathrm{C}$.

\begin{tabular}{cccc}
\hline Drop Height $(\mathrm{cm})$ & Microphone 1 $(\mathrm{dB})$ & Microphone 2 $(\mathrm{dB})$ & Avg. of 1 and 2 $(\mathrm{dB})$ \\
\hline 25 & 124.5 & 127.6 & 126.05 \\
19 & 95.8 & 99.6 & 97.7 \\
30 & 95.8 & 99.5 & 97.65 \\
45 & 128.8 & 130.4 & 129.6 \\
40 & 130.3 & 131.3 & 130.8 \\
20 & 122.3 & 126.8 & 124.55 \\
15 & 98.8 & 96 & 97.4 \\
32 & 127.8 & 130.6 & 129.2 \\
27 & 123.2 & 127.2 & 125.2 \\
21 & 99.6 & 97.9 & 98.75 \\
12 & 99.1 & 95.4 & 97.25 \\
34 & 101.5 & 102.2 & 101.85 \\
43 & 126.7 & 129.7 & 128.2 \\
11 & 99 & 95.7 & 97.35 \\
40 & 128 & 130.5 & 129.25 \\
\hline
\end{tabular}

Table S10. Impact sensitivity test acoustic data on $\left[\mathrm{Ni}(\mathrm{Htrz})_{3}\right]_{n}\left[\mathrm{ClO}_{4}\right]_{2 n}(\mathbf{2})$ at $75{ }^{\circ} \mathrm{C}$.

\begin{tabular}{cccc}
\hline Drop Height $(\mathrm{cm})$ & Microphone 1 $(\mathrm{dB})$ & Microphone 2 $(\mathrm{dB})$ & Avg. of 1 and 2 $(\mathrm{dB})$ \\
\hline 20 & 123.9 & 128 & 125.95 \\
14 & 123 & 127.2 & 125.1 \\
10 & 98.3 & 95.8 & 97.05 \\
13 & 99.9 & 102.2 & 101.05 \\
25 & 131.4 & 134.2 & 132.8 \\
20 & 124.9 & 127.5 & 126.2 \\
15 & 131.3 & 133.1 & 132.2 \\
12 & 128.5 & 130.6 & 129.55 \\
10 & 129 & 131.4 & 130.2 \\
10 & 97.3 & 99.1 & 98.2 \\
12 & 129.2 & 131.4 & 130.3 \\
10 & 127.5 & 130.8 & 129.15 \\
8 & 98.4 & 99.1 & 98.75 \\
15 & 128.6 & 129.5 & 129.05 \\
11 & 125.3 & 127.2 & 126.25 \\
\hline
\end{tabular}




\section{Theoretical Results}

\section{Optimized Cartesian coordinates}

1, $\left[\mathrm{Fe}(\mathrm{Htrz})_{6}\right]^{2+} \mathrm{HS}$

$262.592098-0.3936510 .165178$

$71.7176120 .796562-1.456019$

$72.4521731 .739445-2.147882$

$60.4938900 .789122-1.939583$

$74.313181-0.859472-1.119958$

$64.988973-0.076379-1.933095$

$74.899772-2.108564-1.126165$

$73.568265-1.5296371 .767131$

$63.070928-2.4403412 .576242$

$74.906501-1.3667372 .066911$

$70.841004-0.0658281 .468677$

60.1586391 .0292081 .726754

$70.269964-1.1102642 .166170$

$71.582158-2.194394-0.616011$

$70.244419-2.168220-0.952460$

$62.041818-3.408238-0.831937$

73.4698261 .4585340 .958701

64.6834551 .6833661 .414416

72.7642172 .6441350 .987430

$61.6441582 .274621-3.025353$

63.5797993 .5520151 .457071

$65.918621-2.037854-1.942821$

$65.174468-2.1900163 .046535$

$6-0.063421-3.373558-1.356200$

$6-0.746780-0.6115432 .820481$

$16.595681-2.842984-2.179606$

$76.016106-0.780496-2.469955$

$16.134017-2.3170273 .521888$

$74.052122-2.8870043 .398502$

10.3951392 .0119631 .349775

$7-0.8561200 .7282452 .574769$

$1-0.2944060 .125579-1.620230$

$70.4024051 .710423-2.930136$

$1-1.035389-3.698697-1.691240$

$71.033315-4.186611-1.297235$

$13.065681-3.718640-0.693352$

1 1.087567 -5.162495-1.557924

$13.966700-3.5828374 .127724$

$12.037490-2.7486502 .601483$

$16.703017-0.445455-3.132626$

$14.7388210 .949488-2.155690$ 
$11.8992283 .049598-3.730460$

$1-0.4127781 .925808-3.489003$

15.4474250 .9315991 .539811

74.7970302 .9960021 .735495

13.3455724 .5933771 .610090

15.6112203 .4617332 .114204

$1-1.5420581 .3671462 .954963$

$1-1.413822-1.1607553 .465547$

1, $\left[\mathrm{Fe}(\mathrm{Htrz})_{6}\right]^{2+} \mathrm{LS}$

$262.578368-0.4009920 .167775$

$71.8711950 .536513-1.497934$

$71.856008-0.043549-2.750961$

$61.2981691 .716181-1.587175$

$74.347431-0.797829-0.762955$

$64.547540-1.164993-2.009176$

$75.567318-0.793541-0.115956$

$73.285543-1.3385001 .833484$

$63.858650-2.5181301 .922707$

$73.300614-0.7584933 .086538$

$70.809294-0.0041321 .098482$

60.6091480 .3630222 .344701

$7-0.410567-0.0083470 .451428$

$71.845005-2.159990-0.554523$

$72.607784-3.301888-0.699491$

$60.642713-2.409840-1.023574$

73.3117291 .3580040 .890092

64.5139991 .6078141 .359223

72.5489802 .4999281 .035001

$61.2791310 .806707-3.556136$

63.3169123 .4027111 .582085

$66.464060-1.153270-0.993728$

$63.877505-1.6087403 .891711$

$61.839838-4.204703-1.246502$

$6-1.3073300 .3514041 .329170$

$17.522422-1.246501-0.810966$

$75.869236-1.397229-2.196449$

$14.044344-1.4633684 .946819$

$74.245056-2.7278093 .204357$

11.3762380 .4483973 .095960

$7-0.7125470 .5953042 .531924$

$11.1732412 .407374-0.770621$

$70.9116991 .925831-2.868810$

$12.129742-5.213857-1.491010$

$70.597053-3.688860-1.468447$

$1-0.175516-1.709500-1.034968$ 
$1-0.198186-4.158636-1.878081$

$14.708517-3.5478993 .569801$

$13.983665-3.2092851 .106137$

$16.316329-1.689417-3.054135$

$13.780422-1.250428-2.760401$

$11.1122200 .661301-4.611229$

$10.4482742 .745937-3.234262$

15.3322130 .9074571 .370649

74.5596672 .8868281 .804112

13.0270224 .4118731 .826580

15.3548933 .3565792 .213802

$1-1.1596640 .8874903 .389598$

$1-2.3656800 .4446871 .146364$

2, $\left[\mathrm{Ni}(\mathrm{Htrz})_{6}\right]^{2+} \mathrm{HS}$

$282.578406-0.4009650 .167770$

$71.7268230 .752460-1.381838$

$72.4545321 .707627-2.057470$

$60.5025960 .743634-1.863202$

$74.264572-0.810404-1.034759$

$64.956484-0.009638-1.816127$

$74.834740-2.063890-1.079770$

$73.516096-1.5084631 .701082$

$63.016760-2.4191112 .508489$

$74.856090-1.3605771 .985564$

$70.890865-0.0856601 .396387$

60.2176991 .0188651 .635965

$70.297210-1.1176012 .089988$

$71.612489-2.131761-0.558912$

$70.274311-2.130387-0.887069$

$62.089862-3.340734-0.761458$

73.4600551 .3782920 .884559

64.6871541 .6012601 .302907

72.7535412 .5590420 .955360

$61.6440502 .250913-2.928248$

63.5791063 .4638001 .413748

$65.859431-1.981595-1.888164$

$65.127436-2.1914732 .958332$

$6-0.018054-3.343949-1.277015$

$6-0.722166-0.6038082 .728077$

$16.526780-2.788181-2.146281$

$75.976295-0.709775-2.372469$

$16.091412-2.3286813 .421461$

$74.003333-2.8792493 .317603$

10.4748741 .9952741 .257169

$7-0.8127520 .7345902 .470890$ 
$1-0.2802630 .070369-1.551893$

$70.4067451 .678944-2.840814$

$1-0.986755-3.686160-1.604231$

$71.090514-4.139288-1.212408$

$13.119770-3.630527-0.626134$

$11.159117-5.117273-1.461372$

$13.917468-3.5773074 .044576$

$11.980567-2.7162532 .541290$

$16.669537-0.361979-3.021775$

$14.7196941 .024818-2.008111$

$11.8966523 .037130-3.621515$

$1-0.4098301 .895577-3.397073$

15.4561850 .8507381 .394203

74.8057352 .9086941 .643901

13.3447954 .5013991 .589951

15.6296533 .3702572 .006207

$1-1.4963691 .3844872 .836193$

$1-1.403543-1.1418593 .367441$

2, $\left[\mathrm{Ni}(\mathrm{Htrz})_{6}\right]^{2+} \mathrm{LS}$

$282.634195-0.3576830 .629324$

$70.5392150 .647223-3.382453$

$71.8417870 .760092-3.832978$

$6-0.2189971 .345968-4.192924$

$74.123141-0.498097-0.533579$

$64.183779-0.345985-1.844298$

$75.369965-0.785404-0.039550$

$73.765336-1.8888202 .663391$

$63.465617-3.1425852 .898976$

$74.840052-1.5649073 .471299$

$71.090424-0.1313001 .708204$

60.5130130 .9826392 .117449

$70.405681-1.2109012 .209379$

$71.763590-1.754627-0.309221$

$71.944202-3.0701270 .034849$

$60.911107-1.667580-1.313161$

73.4700221 .1232691 .466004

64.4998711 .1389372 .289596

73.0912822 .4116701 .162138

$61.8204471 .524782-4.898586$

63.9145063 .1880231 .819525

$66.166938-0.813280-1.074694$

$65.150940-2.6314424 .164233$

$61.185829-3.758962-0.776791$

$6-0.571707-0.7176522 .922830$

$17.224383-1.020519-1.044155$ 
$75.469680-0.543552-2.219668$

$15.946165-2.7108424 .888136$

$74.310762-3.6542663 .838191$

10.8319051 .9838141 .874933

$7-0.5439240 .6495132 .891837$

$1-1.2884581 .464028-4.121131$

$70.5441211 .918036-5.164497$

$11.086915-4.832384-0.781180$

$70.525559-2.925013-1.634154$

$10.597122-0.769843-1.843365$

$1-0.117988-3.182778-2.370687$

$14.315998-4.5924004 .214440$

$12.680280-3.6956292 .412358$

$15.832423-0.497162-3.162657$

$13.373553-0.075450-2.518824$

$12.6714681 .813656-5.494537$

$10.2294162 .505904-5.925193$

14.9806170 .2665772 .731752

74.8052572 .4359792 .531866

13.9026364 .2660001 .807841

15.5456392 .7700843 .135173

$1-1.1807471 .2887403 .349974$

$1-1.303320-1.2941283 .465591$

Table S11. Calculated IR peaks and intensities.

\begin{tabular}{|c|c|c|c|c|c|}
\hline \multicolumn{2}{|c|}{ Model 1, $\left[\mathrm{Fe}(\mathrm{Htrz})_{6}\right]^{2+}, \mathrm{LS}$} & \multicolumn{2}{|c|}{ Model 1, $\left[\mathrm{Fe}(\mathrm{Htrz})_{6}\right]^{2+}, \mathrm{HS}$} & \multicolumn{2}{|c|}{ Model 2, $\left[\mathrm{Fe}(\mathrm{Htrz})_{6}\right]^{2+}, \mathrm{HS}$} \\
\hline Freq. $\left(\mathrm{cm}^{-1}\right)$ & $\begin{array}{l}\text { Intensity }\left(10^{-}\right. \\
\left.40 \mathrm{esu}^{2} \mathrm{~cm}^{2}\right)\end{array}$ & Freq. $\left(\mathrm{cm}^{-1}\right)$ & $\begin{array}{l}\text { Intensity }\left(10^{-}\right. \\
\left.40 \mathrm{esu}^{2} \mathrm{~cm}^{2}\right)\end{array}$ & Freq. $\left(\mathrm{cm}^{-1}\right)$ & $\begin{array}{l}\text { Intensity (10- } \\
\left.{ }^{40} \mathrm{esu}^{2} \mathrm{~cm}^{2}\right)\end{array}$ \\
\hline 39.32567 & 19.77813 & 25.9692 & 0.030455 & 34.74973 & 1.602283 \\
\hline 43.22996 & 93.3442 & 34.76411 & 98.66518 & 35.86856 & 43.90742 \\
\hline 43.50064 & 93.0969 & 35.46688 & 93.89585 & 36.14914 & 44.31483 \\
\hline 67.53624 & $1.04 \mathrm{E}-05$ & 51.55894 & 258.8812 & 61.48855 & 269.4259 \\
\hline 72.26113 & $1.77 \mathrm{E}-05$ & 52.12422 & 154.4083 & 61.85056 & 261.6732 \\
\hline 72.55167 & $3.32 \mathrm{E}-05$ & 54.45621 & 84.60695 & 63.44031 & 0.29231 \\
\hline 79.19363 & 271.4475 & 58.66343 & 127.7216 & 69.49372 & 158.8508 \\
\hline 79.49431 & 269.6266 & 59.52408 & 171.4975 & 69.58813 & 673.0294 \\
\hline 81.2501 & 0.000199 & 63.5342 & 1222.697 & 70.10761 & 667.3074 \\
\hline 87.2726 & 1767.567 & 72.83945 & 885.9713 & 73.16815 & 1328.457 \\
\hline 104.1801 & 834.4007 & 87.88222 & 226.7946 & 96.88062 & 0.05099 \\
\hline 111.0718 & $1.92 \mathrm{E}-05$ & 88.10591 & 376.414 & 104.043 & 317.0066 \\
\hline 111.2141 & $9.39 \mathrm{E}-05$ & 89.87618 & 18.38133 & 104.1659 & 333.0224 \\
\hline 140.769 & 719.7173 & 95.97651 & 195.9411 & 119.9006 & 113.605 \\
\hline 140.7927 & 722.1819 & 99.9484 & 69.34374 & 120.0668 & 121.242 \\
\hline 171.7254 & $2.86 \mathrm{E}-06$ & 115.1095 & 198.8159 & 132.1788 & 28.24166 \\
\hline
\end{tabular}




\begin{tabular}{|c|c|c|c|c|c|}
\hline 171.8567 & $3.01 \mathrm{E}-06$ & 119.6037 & 92.37881 & 132.6885 & 29.15399 \\
\hline 184.451 & $8.01 \mathrm{E}-07$ & 123.0405 & 507.0595 & 140.9758 & 374.8634 \\
\hline 185.9063 & $2.21 \mathrm{E}-07$ & 129.2787 & 170.3954 & 149.2833 & 224.7441 \\
\hline 186.0758 & $1.1 \mathrm{E}-06$ & 130.6637 & 115.9958 & 149.3375 & 225.366 \\
\hline 204.0662 & 215.0747 & 137.9847 & 64.24784 & 180.5393 & 6.993918 \\
\hline 204.2375 & 215.2227 & 146.007 & 313.8789 & 180.5495 & 5.42064 \\
\hline 206.4582 & $3.16 \mathrm{E}-05$ & 157.703 & 2.316718 & 183.6017 & 0.015917 \\
\hline 225.6957 & 28.37358 & 164.1414 & 98.66218 & 188.3059 & 28.49749 \\
\hline 242.6591 & $4.22 \mathrm{E}-06$ & 166.6351 & 7.650332 & 188.4162 & 26.77638 \\
\hline 242.7495 & $3.92 \mathrm{E}-07$ & 170.1443 & 39.38617 & 194.2482 & 0.001835 \\
\hline 248.7808 & 239.3264 & 170.5085 & 539.263 & 204.7658 & 115.4287 \\
\hline 250.4034 & 22.17985 & 175.5436 & 301.8243 & 204.8564 & 250.7748 \\
\hline 250.4905 & 21.32431 & 181.1879 & 2.669199 & 205.2363 & 247.6432 \\
\hline 254.9515 & $1.26 \mathrm{E}-06$ & 182.2392 & 439.059 & 207.3367 & 0.717198 \\
\hline 371.6027 & 200.6151 & 238.3374 & 1304.741 & 275.0489 & 1025.212 \\
\hline 390.7745 & 151.3437 & 251.301 & 1150.77 & 287.2371 & 659.7096 \\
\hline 390.9648 & 151.0594 & 253.9905 & 967.5434 & 287.2967 & 659.4722 \\
\hline 613.8076 & $1.85 \mathrm{E}-05$ & 614.0393 & 101.5545 & 613.0496 & 73.19284 \\
\hline 613.8668 & $2.34 \mathrm{E}-05$ & 614.1374 & 96.88873 & 613.1074 & 65.79836 \\
\hline 616.0066 & 550.9871 & 616.8983 & 512.461 & 617.9634 & 2167.789 \\
\hline 616.0686 & 549.8137 & 618.6921 & 515.5204 & 618.1666 & 658.3782 \\
\hline 618.4298 & 2725.981 & 619.0242 & 2245.397 & 618.187 & 519.784 \\
\hline 618.9576 & 0.000566 & 621.35 & 7.090256 & 620.644 & 0.300975 \\
\hline 680.99 & 4.19E-06 & 683.4825 & 212.9365 & 684.2161 & 650.4035 \\
\hline 681.8974 & 0.039084 & 684.5655 & 399.6859 & 684.6841 & 4.082851 \\
\hline 681.9067 & 59.47947 & 684.7797 & 212.5506 & 684.7255 & 3.347491 \\
\hline 682.0059 & 59.0899 & 685.0171 & 13.98525 & 686.0895 & 0.038672 \\
\hline 682.0161 & 0.049371 & 685.8768 & 108.3152 & 686.3701 & 109.512 \\
\hline 682.7643 & 411.2319 & 686.3556 & 7.685759 & 686.4678 & 114.9137 \\
\hline 703.3009 & $9.05 \mathrm{E}-08$ & 691.649 & 1.07915 & 692.299 & 0.048786 \\
\hline 703.429 & $1.54 \mathrm{E}-07$ & 692.8473 & 0.46683 & 692.526 & 0.065205 \\
\hline 708.5498 & $1.37 \mathrm{E}-07$ & 694.7765 & 2.690166 & 698.2359 & 3.903275 \\
\hline 711.3151 & 3.308198 & 695.1526 & 0.019571 & 698.3608 & 3.797579 \\
\hline 711.379 & 3.322683 & 696.4281 & 0.120924 & 699.1973 & 0.013075 \\
\hline 719.463 & 6.49679 & 697.8155 & 0.089334 & 700.0129 & 4.475611 \\
\hline 857.8763 & 9.462808 & 852.1926 & 22.35316 & 850.0664 & 0.043787 \\
\hline 857.9044 & 9.39079 & 852.2226 & 0.140326 & 850.2385 & 0.537837 \\
\hline 857.9322 & 0.002158 & 853.5833 & 26.92993 & 851.0902 & 76.34941 \\
\hline 857.9449 & 0.000479 & 853.6861 & 0.905113 & 851.3323 & 16.54618 \\
\hline 858.6343 & $1.81 \mathrm{E}-05$ & 854.2915 & 49.94701 & 851.4407 & 0.345026 \\
\hline 858.8361 & 129.0566 & 855.153 & 1.072753 & 852.3839 & 0.010971 \\
\hline 907.3912 & 0.000872 & 886.2924 & 51.13811 & 885.4084 & 23.49985 \\
\hline 908.1527 & 0.003782 & 886.6863 & 155.8173 & 885.7916 & 29.73069 \\
\hline
\end{tabular}




\begin{tabular}{|c|c|c|c|c|c|}
\hline 908.6323 & 0.025215 & 889.7091 & 37.16822 & 887.6621 & 311.3963 \\
\hline 908.789 & 152.3307 & 889.764 & 206.8806 & 888.1775 & 9.709857 \\
\hline 909.2127 & 154.3934 & 893.2312 & 170.6198 & 889.7059 & 217.5026 \\
\hline 910.8516 & 223.9602 & 895.995 & 133.617 & 890.3412 & 202.6095 \\
\hline 974.2752 & $2.66 \mathrm{E}-07$ & 955.1475 & 26.03102 & 960.5084 & 21.27681 \\
\hline 974.3407 & 7.37E-06 & 958.8122 & 12.7952 & 960.5441 & 21.73465 \\
\hline 974.839 & $2.99 \mathrm{E}-05$ & 959.5717 & 1.474644 & 961.6962 & 0.562843 \\
\hline 975.7943 & 63.20144 & 959.8923 & 6.37963 & 961.7884 & 0.601718 \\
\hline 975.8492 & 62.73515 & 963.674 & 0.150488 & 966.3536 & 1.561833 \\
\hline 976.0236 & 41.68536 & 964.7554 & 0.004045 & 968.3688 & 0.001324 \\
\hline 979.0721 & 5.879889 & 969.1617 & 27.11591 & 970.5041 & 29.97401 \\
\hline 979.1318 & 5.603764 & 969.6628 & 7.354925 & 971.1756 & 13.74605 \\
\hline 979.4402 & $8.83 \mathrm{E}-06$ & 970.0736 & 16.04865 & 971.2615 & 13.26221 \\
\hline 979.4598 & $2.81 \mathrm{E}-05$ & 971.3317 & 7.320301 & 972.5321 & 7.466148 \\
\hline 984.7237 & 12.81422 & 971.3426 & 15.14609 & 972.6423 & 7.325097 \\
\hline 985.4904 & $2.1 \mathrm{E}-06$ & 971.4783 & 0.010472 & 972.8339 & 0.116164 \\
\hline 1050.175 & 30.78647 & 1042.194 & 121.5605 & 1049.165 & 127.5977 \\
\hline 1050.727 & $8.34 \mathrm{E}-05$ & 1042.973 & 56.14873 & 1050.859 & 4.366423 \\
\hline 1050.81 & $9.34 \mathrm{E}-05$ & 1043.609 & 0.963482 & 1050.963 & 2.517885 \\
\hline 1052.495 & 78.53793 & 1047.371 & 207.4551 & 1055.189 & 188.5694 \\
\hline 1052.645 & 77.86842 & 1048.247 & 200.241 & 1055.205 & 189.2157 \\
\hline 1054.966 & $2.38 \mathrm{E}-05$ & 1049.21 & 31.50112 & 1056.25 & 0.112978 \\
\hline 1123.361 & 176.5935 & 1117.413 & 181.5721 & 1120.127 & 182.3401 \\
\hline 1123.383 & 176.5836 & 1117.751 & 0.976491 & 1120.158 & 180.1572 \\
\hline 1123.64 & $3.03 \mathrm{E}-05$ & 1117.769 & 82.44657 & 1120.769 & 4.118721 \\
\hline 1123.674 & 0.000595 & 1118.363 & 103.7025 & 1120.793 & 1.672444 \\
\hline 1124.841 & $6.88 \mathrm{E}-06$ & 1120.061 & 1.578487 & 1122.365 & 129.1803 \\
\hline 1126.612 & 99.12091 & 1120.151 & 131.2421 & 1122.521 & 0.086867 \\
\hline 1138.097 & 108.3158 & 1136.709 & 37.53974 & 1136.832 & 39.19543 \\
\hline 1140.899 & $1.2 \mathrm{E}-06$ & 1139.084 & 0.424048 & 1140.424 & 0.00082 \\
\hline 1145.908 & 0.00022 & 1139.834 & 61.74783 & 1142.471 & 34.37502 \\
\hline 1145.969 & 0.00041 & 1141.21 & 0.361551 & 1142.567 & 13.2923 \\
\hline 1146.27 & 230.1644 & 1141.754 & 155.7564 & 1143.413 & 150.1384 \\
\hline 1146.291 & 230.2598 & 1141.783 & 103.6104 & 1143.465 & 129.7621 \\
\hline 1241.812 & $1.76 \mathrm{E}-07$ & 1232.766 & 3.141684 & 1233.124 & 2.542246 \\
\hline 1243.552 & 0.324843 & 1234.537 & 0.053188 & 1235.503 & 0.114471 \\
\hline 1247.473 & $1.64 \mathrm{E}-05$ & 1236.556 & 0.041295 & 1238.087 & 0.073747 \\
\hline 1247.618 & $1.08 \mathrm{E}-06$ & 1236.979 & 22.56165 & 1238.202 & 9.022604 \\
\hline 1249.038 & 41.67877 & 1237.425 & 0.922848 & 1238.562 & 18.44574 \\
\hline 1249.153 & 41.69991 & 1237.575 & 26.70087 & 1238.623 & 27.97753 \\
\hline 1328.152 & $9.07 \mathrm{E}-06$ & 1317.197 & 31.05087 & 1320.354 & 43.15302 \\
\hline 1329.208 & $6.17 \mathrm{E}-06$ & 1318.16 & 60.70721 & 1320.498 & 38.8399 \\
\hline 1329.262 & $1.24 \mathrm{E}-05$ & 1318.312 & 45.41836 & 1320.513 & 36.20508 \\
\hline
\end{tabular}




\begin{tabular}{|c|c|c|c|c|c|}
\hline 1330.015 & 42.63097 & 1318.677 & 12.76354 & 1322.457 & 26.05252 \\
\hline 1330.064 & 42.75715 & 1319.482 & 37.07483 & 1322.523 & 27.76667 \\
\hline 1331.412 & 18.7483 & 1320.498 & 10.33902 & 1323.724 & 0.130632 \\
\hline 1441.486 & 0.000226 & 1429.167 & 20.50055 & 1434.96 & 48.87973 \\
\hline 1441.513 & $4.33 \mathrm{E}-05$ & 1429.379 & 80.81283 & 1434.988 & 46.11863 \\
\hline 1442.139 & 187.8039 & 1429.783 & 165.9441 & 1435.756 & 128.8133 \\
\hline 1442.165 & 187.7412 & 1431.227 & 81.50244 & 1435.809 & 124.6931 \\
\hline 1443.091 & 13.91679 & 1431.862 & 41.19021 & 1436.26 & 31.20612 \\
\hline 1443.831 & $6.43 \mathrm{E}-06$ & 1432.73 & 13.992 & 1437.514 & 0.032531 \\
\hline 1536.063 & 221.0474 & 1524.746 & 93.31596 & 1527.679 & 62.75807 \\
\hline 1536.099 & 221.0046 & 1524.907 & 72.00283 & 1527.721 & 58.66039 \\
\hline 1536.279 & $7.36 \mathrm{E}-05$ & 1526.662 & 145.7318 & 1530.388 & 166.1433 \\
\hline 1536.319 & 0.001373 & 1526.931 & 160.4988 & 1530.449 & 162.4369 \\
\hline 1537.672 & $2.62 \mathrm{E}-05$ & 1528.735 & 8.171541 & 1531.63 & 3.241738 \\
\hline 1539.848 & 5.813171 & 1530.51 & 0.984706 & 1533.758 & 0.056522 \\
\hline 1552.321 & 155.1013 & 1548.404 & 217.8039 & 1548.346 & 208.9167 \\
\hline 1552.533 & 0.000327 & 1550.182 & 48.66729 & 1550.802 & 8.011428 \\
\hline 1555.087 & 0.001949 & 1550.571 & 10.68523 & 1550.808 & 44.6565 \\
\hline 1555.1 & 0.00072 & 1550.852 & 86.91207 & 1550.819 & 39.21486 \\
\hline 1555.444 & 159.1675 & 1551.547 & 81.99349 & 1552.218 & 124.1205 \\
\hline 1555.449 & 159.2882 & 1551.99 & 140.7332 & 1552.27 & 121.4209 \\
\hline 3269.636 & 0.001095 & 3262.928 & 59.89514 & 3268.786 & 5.629782 \\
\hline 3269.651 & 5.631963 & 3263.186 & 1.754068 & 3269.21 & 21.51476 \\
\hline 3269.942 & 0.000765 & 3267.153 & 41.74048 & 3269.97 & 62.4638 \\
\hline 3269.968 & 0.115178 & 3267.507 & 2.342346 & 3270.013 & 85.49356 \\
\hline 3269.982 & 75.34742 & 3267.653 & 82.22486 & 3270.321 & 17.43258 \\
\hline 3270.007 & 74.40468 & 3267.847 & 2.404166 & 3270.467 & 29.40416 \\
\hline 3274.918 & 0.000476 & 3275.258 & 1.06026 & 3276.585 & 5.740375 \\
\hline 3275.129 & 3.815883 & 3275.299 & 2.528268 & 3276.628 & 0.696427 \\
\hline 3276.211 & 0.021318 & 3275.573 & 5.559031 & 3276.652 & 0.76024 \\
\hline 3276.254 & 72.50941 & 3275.605 & 0.704837 & 3276.709 & 0.503127 \\
\hline 3276.347 & 1.110648 & 3275.877 & 2.232871 & 3276.756 & 0.637279 \\
\hline 3276.382 & 72.71632 & 3275.917 & 2.696022 & 3276.786 & 1.110666 \\
\hline 3628.957 & 0.018215 & 3610.66 & 86.7729 & 3611.594 & 177.2823 \\
\hline 3629.057 & 335.4792 & 3610.785 & 290.1478 & 3611.624 & 64.75152 \\
\hline 3629.062 & 2.354068 & 3610.965 & 45.60885 & 3611.716 & 256.4567 \\
\hline 3629.141 & 421.254 & 3611.23 & 286.7141 & 3611.894 & 193.9551 \\
\hline 3629.278 & 411.149 & 3611.279 & 268.7014 & 3611.979 & 313.8799 \\
\hline 3630.503 & 0.000509 & 3612.139 & 3.698761 & 3612.961 & 2.034404 \\
\hline
\end{tabular}



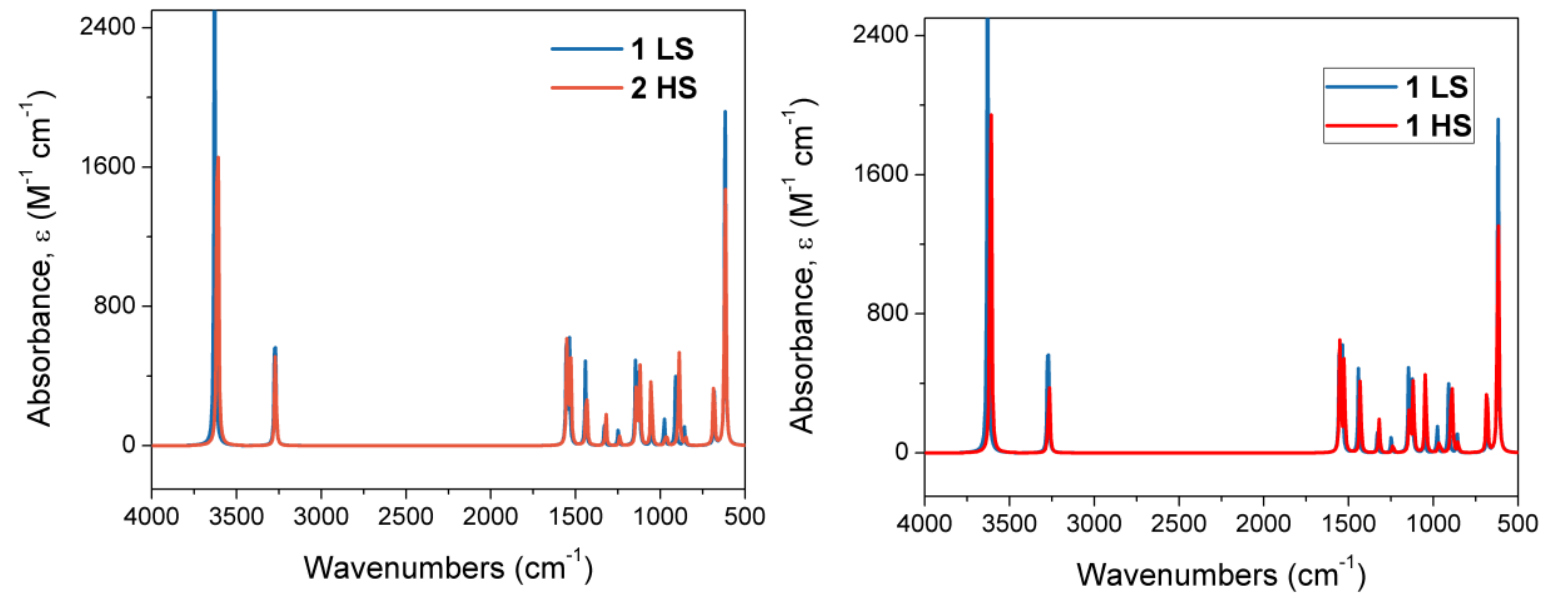

Figure S23. DFT calculated IR spectra for (left) 1 LS and 2 HS and (right) 1 LS and 1 HS.

Table S12. Molecular orbitals of 1 and 2 in LS and HS states.

$\mathrm{d}_{\mathrm{xy}}$




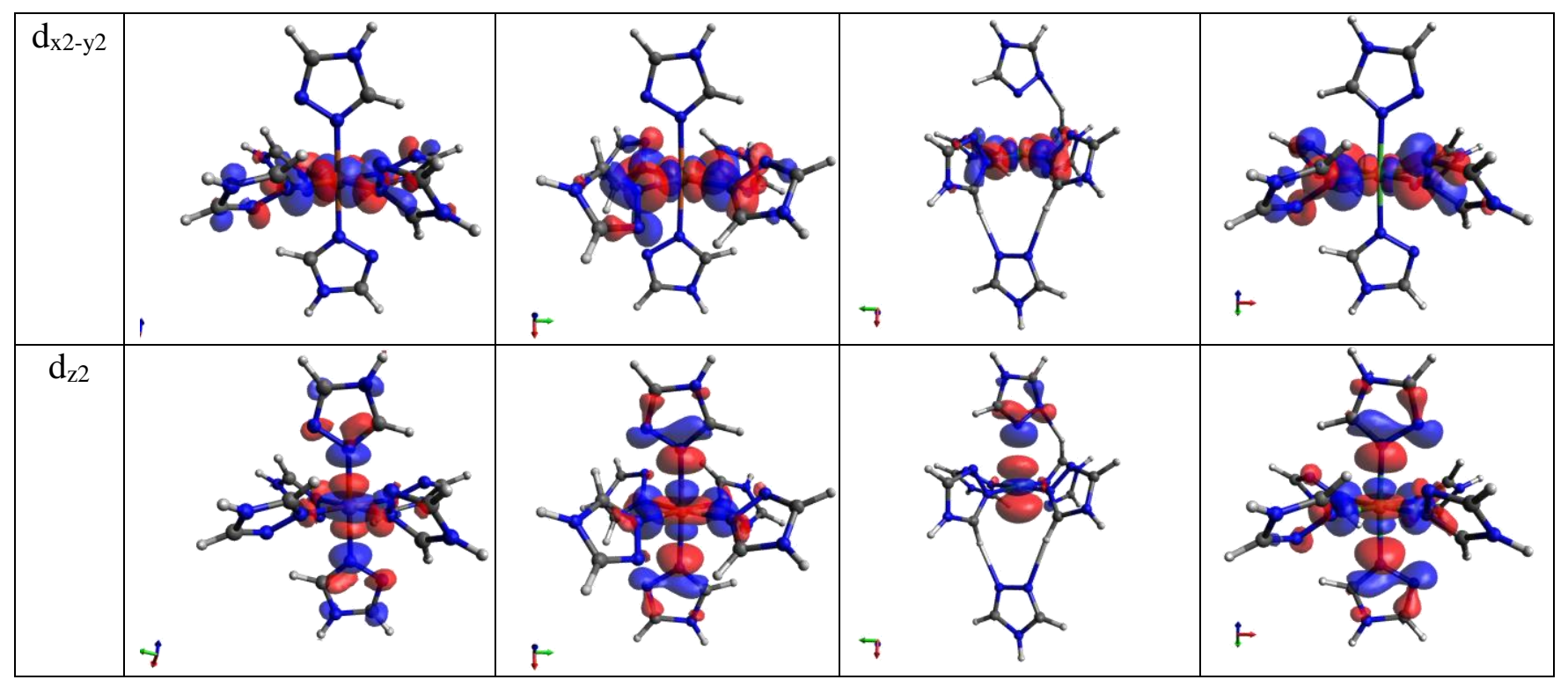

